\title{
Some Coincidence and Common Fixed Point Results in Fuzzy Metric Space with an Application to Differential Equations
}

\author{
Saif Ur Rehman ${ }^{D},{ }^{1}$ Iqra Shamas, ${ }^{1}$ Naeem Jan, ${ }^{1}$ Abdu Gumaei ${ }^{D},{ }^{2}$ \\ and Mabrook Al-Rakhami ${ }^{3}{ }^{3}$ \\ ${ }^{1}$ Department of Mathematics, Gomal University, Dera Ismail Khan 29050, Pakistan \\ ${ }^{2}$ Computer Science Department, Faculty of Applied Sciences, Taiz University, Taiz 6803, Yemen \\ ${ }^{3}$ STC's Artificial Intelligence Chair, Department of Information Systems, College of Computer and Information Sciences, \\ King Saud University, Riyadh 11543, Saudi Arabia
}

Correspondence should be addressed to Abdu Gumaei; abdugumaei@taiz.edu.ye

and Mabrook Al-Rakhami; malrakhami@ksu.edu.sa

Received 16 July 2021; Revised 20 September 2021; Accepted 25 September 2021; Published 29 October 2021

Academic Editor: Andreea Fulga

Copyright (c) 2021 Saif Ur Rehman et al. This is an open access article distributed under the Creative Commons Attribution License, which permits unrestricted use, distribution, and reproduction in any medium, provided the original work is properly cited.

\begin{abstract}
In this paper, we study some coincidence point and common fixed point theorems in fuzzy metric spaces by using three-selfmappings. We prove the uniqueness of some coincidence point and common fixed point results by using the weak compatibility of three-self-mappings. In support of our results, we present some illustrative examples for the validation of our work. Our results extend and improve many results given in the literature. In addition, we present an application of fuzzy differential equations to support our work.
\end{abstract}

\section{Introduction}

Zadeh [1] introduced the concept of fuzzy sets which is defined as "a set contracted from a function having a domain is a nonempty set $\Omega$ and range in $[0,1]$ is called a fuzzy set, that is if $G: \Omega \longrightarrow[0,1]$." In 1975, Kramosil and Michalek [2] introduced the notion of fuzzy metric (FM) space, and they compared the concept of fuzzy metric with the statistical metric space and proved that both the conceptions are equivalent in some cases. Later on, George and Veeramani [3] modify the concept of Kramosil and Michalek [2] and proved that every metric induces a fuzzy metric. They proved some basic properties and Baire's theorem for fuzzy metric spaces. In 1988, Grabiec [4] used the concept of Kramosil and Michalek [2] and proved fixed point (FP) theorems of "Banach and Edelstein contraction mapping theorems on complete and compact FM spaces, respectively." Gregori and Sapena [5], Imdad and Ali [6], Mihet [7, 8], Bari and Vetro [9], and Som [10] proved some FP and common fixed point (CFP) theorems in FM spaces. Aliouche et al. [11] and Rao et al. [12] established some related FP in FM spaces.

Hadzic and Pap [13] established some multivalued FP results in probabilistic metric spaces with an application in FM spaces. Later on, Kiany and Amini-Haradi [14] obtained some FP and end-point theorems for set-valued contractive type mappings in FM spaces. In [15], Beg et al. proved some FP results for self-mappings satisfying an implicit relation in a complete FM space. Rolden et al. [16] established some new FP theorems in FM spaces, while in [17], Jeli et al. presented some results by using cyclic $(\psi, \phi)$-contractions in Kaleva-Seikkala's type FM spaces. Later on, Li et al. [18] proved some strong coupled FP theorems in complete FM spaces with an integral type of application. The concept of rational type fuzzy-contraction is given by Rehman et al. [19]. They proved some unique FP theorems with the application of nonlinear integral in FM spaces. Shamas et al. [20] proved some unique FP results in FM spaces with an application to Fredholm integral equations. Recently, Jabeen et al. 
[21] presented the concept of weakly compatible selfmappings in fuzzy cone metric spaces, and they proved some coincidence point and CFP theorems in the said space with integral type application. Some more coincidence points, coupled coincidence points, and CFP findings in deferent types of metric spaces can be found in (e.g., see [22-35] the references therein).

In this paper, we establish some unique coincidence points and CFP theorems in FM space by using the concept of Gregori and Sapena [5] and Jabeen et al. [21]. We establish some generalized fuzzy-contraction results for weakly compatible three self-mappings in FM spaces without the assumption that the "fuzzy contractive sequences are Cauchy." We present some illustrative examples and an application of fuzzy differential equation to support our work. By using this concept, researchers can prove more coincidence points and CFP results for different contractive type mappings in FM spaces with the application of integral operators.

The layout of this paper is as follows: Section 2 consists of preliminary concepts. While Section 3 deals with the main results of this paper in which we shall prove unique coincidence points and CFP theorems by using weaklycompatible three self-mappings in FM spaces with some illustrative examples. In Section 4, we establish an application of fuzzy differential equations to support our main work. While in the last section, that is, Section 5 is the conclusion part of our paper.

\section{Preliminaries}

In this section, we recall some basic definitions related to our main work such as continuous $t$-norm, FM space, Cauchy sequence, fuzzy-contraction, and weak-compatible mappings. The concept of continuous $t$-norm is given by Schweizer and Sklar [36].

Definition 1 (see [36]). An operation $*:[0,1] \times[0,1] \longrightarrow$ $[0,1]$ is known as a continuous $t$-norm if it fulfils the following axioms;

(1) * is associative, commutative, and continuous.

(2) $1 * \rho_{1}=\rho_{1}$ and $\rho_{1} * \rho_{2} \leq \rho_{3} * \rho_{4}$, whenever $\rho_{1} \leq \rho_{3}$ and $\rho_{2} \leq \rho_{4}$, for each $\rho_{1}, \rho_{2}, \rho_{3}, \rho_{4} \in[0,1]$.

The basic continuous $t$-norms are (see; [36]): the minimum, the product, and the Lukasiewicz $t$-norms are defined respectively as follows;

$$
\begin{aligned}
\rho_{1} * \rho_{2} & =\min \left\{\rho_{1}, \rho_{2}\right\}, \rho_{1} * \rho_{2}=\rho_{1} \rho_{2} \text { and } \rho_{1} * \rho_{2} \\
& =\max \left\{\rho_{1}+\rho_{2}-1,0\right\} .
\end{aligned}
$$

Definition 2 (see [3]). A 3-tuple $\left(\Omega, M_{F}, *\right)$ is said to be a FM space if $\Omega$ is an arbitrary set, $*$ is a continuous $t$-norm, and $M_{F}$ is a fuzzy set on $\Omega \times \Omega \times(0, \infty)$ satisfying the following;

(i) $M_{F}(\xi, \eta, t)>0$ and $M_{F}(\xi, \eta, t)=1 \Leftrightarrow \xi=\eta$.

(ii) $M_{F}(\xi, \eta, t)=M_{F}(\eta, \xi, t)$. (iii) $M_{F}(\xi, \zeta, t) * M_{F}(\zeta, \eta, s) \leq M_{F}(\xi, \eta, t+s)$.

(iv) $M_{F}(\xi, \eta, \cdot):(0, \infty) \longrightarrow[0,1]$ is continuous

for all $\xi, \zeta, \eta \in \Omega$ and $t, s>0$.

Definition 3 (see $[3,5])$. Let $\left(\Omega, M_{F}, *\right)$ be a FM space, $\xi \in \Omega$ and $\left\{\xi_{j}\right\}$ be a sequence in $\Omega$. Then

(i) $\left\{\xi_{j}\right\}$ is said to converge to $\xi$ if for $t>0$ and $\varepsilon \in(0$, 1 ), $\exists j_{1} \in \mathbf{N}$ where ( $\mathbf{N}$ represent the set of natural numbers) such that $M_{F}\left(\xi_{j}, \xi, t\right)>1-\varepsilon, \forall j \geq j_{1}$. We represent this by $\lim _{j \longrightarrow \infty} \xi_{j}=\xi$ or $\xi_{j} \longrightarrow \xi$ as $j \longrightarrow \infty$.

(ii) $\left\{w_{i}\right\}$ is said to be a Cauchy sequence, if for $t>0$ and $\varepsilon \in(0,1), \exists j_{1} \in \mathbf{N}$ such that $M_{F}\left(\xi_{j}, \xi_{m}, t\right)>1-\varepsilon$, $\forall j, m \geq j_{1}$.

(iii) $\left(\Omega, M_{F}, *\right)$ is complete, if every Cauchy sequence is convergent in $\Omega$.

(iv) $\left\{\xi_{j}\right\}$ is known as a fuzzy-contractive, if there is 0 $<\beta<1$ so that

$$
\begin{aligned}
\frac{1}{M_{F}\left(\xi_{j}, \xi_{j+1}, t\right)}-1 & \leq \beta\left(\frac{1}{M_{F}\left(\xi_{i-1}, \xi_{i}, t\right)}-1\right) \text { for } t \\
& >0, \text { and } j \geq 1
\end{aligned}
$$

Lemma 4 (see [3]). $M_{F}(\xi, \eta, *)$ is nondecreasing $\forall \xi, \eta \in \Omega$.

Lemma 5 (see [3]). Let $\left(\Omega, M_{F}, *\right)$ be a FM space and let a sequence $\left\{\xi_{j}\right\}$ in $\Omega$ converges to a point $\xi \in \Omega$ iff $M_{F}(\xi, \xi, t)$ $\longrightarrow 1$, as $j \longrightarrow \infty$, for $t>0$.

Definition 6 (see [9]). Let $\left(\Omega, M_{F}, *\right)$ be a FM space. The FM $M_{F}$ is triangular if

$$
\frac{1}{M_{F}(\xi, \eta, t)}-1 \leq\left(\frac{1}{M_{F}(\xi, \zeta, t)}-1\right)+\left(\frac{1}{M_{F}(\zeta, \eta, t)}-1\right)
$$

for all $\xi, \zeta, \eta \in \Omega$ and $t>0$.

Note: easily one can prove that a FM $M_{F}$ is triangular if we define a mapping $M_{F}: \Omega \times \Omega \times$ int $(\mathscr{C}) \longrightarrow[0,1]$ by $M_{F}(\xi, \eta, t)=t / t+|\xi-\eta|$, for all $\xi, \eta \in \Omega$, and $t>0$.

Lemma 7 (see [3]). Let $\left(\Omega, M_{F}, *\right)$ be a FM space. Let $\xi \in \Omega$ and $\left\{\xi_{j}\right\}$ be a sequence in $\Omega$. Then $\xi_{j} \longrightarrow \xi$ iff $\lim _{j \longrightarrow \infty} M_{F}\left(\xi_{j}\right.$, $\xi, t)=1$, for $t>0$.

Definition 8 (see [5]). Let $\left(\Omega, M_{F}, *\right)$ be a FM space and $G: \Omega \longrightarrow \Omega$. Then, $G$ is known as a fuzzy-contraction, if 
there is $0<\beta<1$ so that

$$
\frac{1}{M_{F}(G \xi, G \eta, t)}-1 \leq \beta\left(\frac{1}{M_{F}(\xi, \eta, t)}-1\right)
$$

for all $\xi, \eta \in \Omega$ and $t>0$.

Definition 9 (see [37]). Let $G$ and $\ell$ be two self-mappings on a nonempty set $\Omega$ (i.e., $G, \ell: \Omega \longrightarrow \Omega$ ). If $\exists \xi \in \Omega$ and $\xi=$ $G \omega=\ell \omega$ for some $\omega \in \Omega$. Then $\omega$ is called a coincidence point of $G$ and $\ell$, and $\xi$ is called a point of coincidence of the mappings $G$ and $\ell$. The mappings $G$ and $\ell$ are said to be weakly-compatible if they commute at their coincidence point, i.e., $A \omega=\ell \omega$ for some $\omega \in \Omega$, then $A \ell \omega=\ell A \omega$.

Proposition 10 (see [37]). Let $G$ and $\ell$ be weakly-compatible self-mappings on a nonempty set $\Omega$. If $G$ and $\ell$ have a unique point of coincidence such that $\xi=A \omega=\ell \omega$, then $\xi$ is known as the unique CFP of $G$ and $\ell$.

\section{Main Results}

In this section, we present some unique coincidence points and CFP theorems in FM spaces by using weaklycompatible three self-mappings with illustrative examples.

Theorem 11. Let $\left(\Omega, M_{F}, *\right)$ be a FM space in which a FM $M_{F}$ is triangular. Let $G, H, \ell: \Omega \longrightarrow \Omega$ be three selfmappings that satisfy

$$
\begin{aligned}
& \frac{1}{M_{F}(G \xi, H \eta, t)}-1 \\
& \quad \leq \beta_{1}\left(\frac{1}{M_{F}(\ell \xi, \ell \eta, t)}-1\right)+\beta_{2}\left(\left(\frac{1}{M_{F}(\ell \xi, G \xi, t)}-1\right)\right. \\
& \quad+\left(\frac{1}{M_{F}(\ell \eta, H \eta, t)}-1\right)+\left(\frac{1}{M_{F}(\ell \eta, G \xi, t)}-1\right) \\
& \left.\quad+\left(\frac{1}{M_{F}(\ell \xi, H \eta, t)}-1\right)\right) \\
& \quad+\beta_{3} \max \left\{\left(\frac{1}{M_{F}(\ell \xi, G \xi, t)}-1\right),\left(\frac{1}{M_{F}(\ell \eta, H \eta, t)}-1\right),\right. \\
& \left.\quad\left(\frac{1}{M_{F}(\ell \xi, H \eta, t)}-1\right),\left(\frac{1}{M_{F}(\ell \eta, G \xi, t)}-1\right)\right\},
\end{aligned}
$$

for all $\xi, \eta \in \Omega, t>0$, and $\beta_{1}, \beta_{2}, \beta_{3} \in[0,1)$ with $\left(\beta_{1}+4 \beta_{2}+\right.$ $\left.2 \beta_{3}\right)<1$. If $G(\Omega) \cup H(\Omega) \subset \ell(\Omega)$ and $\ell(\Omega)$ is a complete subspace of $\Omega$. Then, $G, H$, and $\ell$ have a unique point of coincidence. In addition, if $(G, \ell)$ and $(H, \ell)$ are weaklycompatible, then, $G, H$, and $\ell$ have a unique CFP.

Proof. Let $\xi_{0} \in \Omega$ be arbitrary and by using the hypothesis $G($ $\Omega) \cup H(\Omega) \subset \ell(\Omega)$. Now, we choose a sequence $\left\{\xi_{j}\right\}$ such that

$$
\ell \xi_{2 j+1}=G \xi_{2 j} \text { and } \ell \xi_{2 j+2}=H \xi_{2 j+1} \text { for all } j \geq 0 \text {. }
$$

Now by the view of (5) and (6), for $t>0$, we have

$$
\begin{aligned}
& \frac{1}{M_{F}\left(\ell \xi_{2 j+1}, \ell \xi_{2 j+2}, t\right)}-1=\frac{1}{M_{F}\left(G \xi_{2 j}, H \xi_{2 j+1}, t\right)} \\
& -1 \leq \beta_{1}\left(\frac{1}{M_{F}\left(\ell \xi_{2 j}, \ell \xi_{2 j+1}, t\right)}-1\right) \\
& +\beta_{2}\left(\left(\frac{1}{M_{F}\left(\ell \xi_{2 j}, G \xi_{2 j}, t\right)}-1\right)\right. \\
& +\left(\frac{1}{M_{F}\left(\ell \xi_{2 j+1}, H \xi_{2 j+1}, t\right)}-1\right)+\left(\frac{1}{M_{F}\left(\ell \xi_{2 j+1}, G \xi_{2 j}, t\right)}-1\right) \\
& \left.+\left(\frac{1}{M_{F}\left(\ell \xi_{2 j}, H \xi_{2 j+1}, t\right)}-1\right)\right) \\
& +\beta_{3} \max \left\{\left(\frac{1}{M_{F}\left(\ell \xi_{2 j}, G \xi_{2 j}, t\right)}-1\right),\left(\frac{1}{M_{F}\left(\ell \xi_{2 j+1}, H \xi_{2 j+1}, t\right)}-1\right),\right. \\
& \left.\left(\frac{1}{M_{F}\left(\ell \xi_{2 j+1}, G \xi_{2 j}, t\right)}-1\right),\left(\frac{1}{M_{F}\left(\ell \xi_{2 j}, H \xi_{2 j+1}, t\right)}-1\right),\right\} \\
& \leq \beta_{1}\left(\frac{1}{M_{F}\left(\ell \xi_{2 j}, \ell \xi_{2 j+1}, t\right)}-1\right)+\beta_{2}\left(\left(\frac{1}{M_{F}\left(\ell \xi_{2 j}, \ell \xi_{2 j+1}, t\right)}-1\right)\right. \\
& +\left(\frac{1}{M_{F}\left(\ell \xi_{2 j+1}, \ell \xi_{2 j+2}, t\right)}-1\right)+\left(\frac{1}{M_{F}\left(\ell \xi_{2 j}, \ell \xi_{2 j+1}, t\right)}-1\right) \\
& \left.+\left(\frac{1}{M_{F}\left(\ell \xi_{2 j+1}, \ell \xi_{2 j+2}, t\right)}-1\right)\right) \\
& +\beta_{3} \max \left\{\left(\frac{1}{M_{F}\left(\ell \xi_{2 j}, \ell \xi_{2 j+1}, t\right)}-1\right)\right. \text {, } \\
& \left.\left(\frac{1}{M_{F}\left(\ell \xi_{2 j+1}, \ell \xi_{2 j+2}, t\right)}-1\right),\left(\frac{1}{M_{F}\left(\ell \xi_{2 j}, \ell \xi_{2 j+2}, t\right)}-1\right)\right\} \text {. }
\end{aligned}
$$

After simplification, for $t>0$, we obtain

$$
\begin{aligned}
& \frac{1}{M_{F}\left(\ell \xi_{2 j+1}, \ell \xi_{2 j+2}, t\right)}-1 \leq\left(\frac{\beta_{1}+2 \beta_{2}}{1-2 \beta_{2}}\right)\left(\frac{1}{M_{F}\left(\ell \xi_{2 j}, \ell \xi_{2 j+1}, t\right)}-1\right) \\
& +\left(\frac{\beta_{3}}{1-2 \beta_{2}}\right) \max \left\{\left(\frac{1}{M_{F}\left(\ell \xi_{2 j}, \ell \xi_{2 j+1}, t\right)}-1\right),\right. \\
& \left.\left(\frac{1}{M_{F}\left(\ell \xi_{2 j+1}, \ell \xi_{2 j+2}, t\right)}-1\right),\left(\frac{1}{M_{F}\left(\ell \xi_{2 j}, \ell \xi_{2 j+2}, t\right)}-1\right)\right\} .
\end{aligned}
$$

Now three possibilities arise:

(i) If $\left(1 / M_{F}\left(\ell \xi_{2 j}, \ell \xi_{2 j+1}, t\right)-1\right)$ is a maximum term in (8) for $t>0$, then after simplification, we get that

$$
\begin{gathered}
\frac{1}{M_{F}\left(\ell \xi_{2 j+1}, \ell \xi_{2 j+2}, t\right)}-1 \leq\left(\frac{\beta_{1}+2 \beta_{2}+\beta_{3}}{1-2 \beta_{2}}\right) \\
\cdot\left(\frac{1}{M_{F}\left(\ell \xi_{2 j}, \ell \xi_{2 j+1}, t\right)}-1\right) \text { for } t>0 .
\end{gathered}
$$


(ii) If $\left(1 / M_{F}\left(\ell \xi_{2 j+1}, \ell \xi_{2 j+2}, t\right)-1\right)$ is a maximum term in (8) for $t>0$, then after simplification, we get that

$$
\begin{gathered}
\frac{1}{M_{F}\left(\ell \xi_{2 j+1}, \ell \xi_{2 j+2}, t\right)}-1 \leq\left(\frac{\beta_{1}+2 \beta_{2}}{1-2 \beta_{2}-\beta_{3}}\right) \\
\cdot\left(\frac{1}{M_{F}\left(\ell \xi_{2 j}, \ell \xi_{2 j+1}, t\right)}-1\right) \text { for } t>0 .
\end{gathered}
$$

(iii) If $\left(1 / M_{F}\left(\ell \xi_{2 j}, \ell \xi_{2 j+2}, t\right)-1\right)$ is a maximum term in (8) for $t>0$, then after simplification, we get that

$$
\begin{gathered}
\frac{1}{M_{F}\left(\ell \xi_{2 j+1}, \ell \xi_{2 j+2}, t\right)}-1 \leq\left(\frac{\beta_{1}+2 \beta_{2}+\beta_{3}}{1-2 \beta_{2}-\beta_{3}}\right) \\
\cdot\left(\frac{1}{M_{F}\left(\ell \xi_{2 j}, \ell \xi_{2 j+1}, t\right)}-1\right) \text { for } t>0 .
\end{gathered}
$$

Assume that $\lambda:=\max \left\{\left(\beta_{1}+2 \beta_{2}+\beta_{3}\right) /\left(1-2 \beta_{2}\right),\left(\beta_{1}+\right.\right.$ $\left.\left.2 \beta_{2}\right) /\left(1-2 \beta_{2}-\beta_{3}\right),\left(\beta_{1}+2 \beta_{2}+\beta_{3}\right) /\left(1-2 \beta_{2}-\beta_{3}\right)\right\}<1$,

then from (9), (10), and (11), we get that

$\frac{1}{M_{F}\left(\ell \xi_{2 j+1}, \ell \xi_{2 j+2}, t\right)}-1 \leq \lambda\left(\frac{1}{M_{F}\left(\ell \xi_{2 j}, \ell \xi_{2 j+1}, t\right)}-1\right)$ for $t>0$.

Similarly, again from (5) and (6), for $t>0$,

$$
\begin{aligned}
& \frac{1}{M_{F}\left(\ell \xi_{2 j+2}, \ell \xi_{2 j+3}, t\right)}-1=\frac{1}{M_{j}\left(G \xi_{2 j+2}, H \xi_{2 j+1}, t\right)} \\
& \quad-1 \leq \beta_{1}\left(\frac{1}{M_{F}\left(\ell \xi_{2 j+2}, \ell \xi_{2 j+1}, t\right)}-1\right) \\
& +\beta_{2}\left(\left(\frac{1}{M_{F}\left(\ell \xi_{2 j+2}, G \xi_{2 j+2}, t\right)}-1\right)+\left(\frac{1}{M_{F}\left(\ell \xi_{2 j+1}, H \xi_{2 j+1}, t\right)}-1\right)\right. \\
& \left.+\left(\frac{1}{M_{F}\left(\ell \xi_{2 j+1}, G \xi_{2 j+2}, t\right)}-1\right)+\left(\frac{1}{M_{F}\left(\ell \xi_{2 j+2}, H \xi_{2 j+1}, t\right)}-1\right)\right) \\
& +\beta_{3} \max \left\{\left(\frac{1}{M_{F}\left(\ell \xi_{2 j+2}, G \xi_{2 j+2}, t\right)}-1\right),\right. \\
& +\left(\frac{1}{M_{F}\left(\ell \xi_{2 j+1}, H \xi_{2 j+1}, t\right)}-1\right),\left(\frac{1}{M_{F}\left(\ell \xi_{2 j+1}, G \xi_{2 j+2}, t\right)}-1\right) \\
& \left.+\left(\frac{1}{M_{F}\left(\ell \xi_{2 j+2}, H \xi_{2 j+1}, t\right)}-1\right)\right\} \leq \beta_{1}\left(\frac{1}{M_{F}\left(\ell \xi_{2 j+2}, \ell \xi_{2 j+1}, t\right)}-1\right) \\
& +\beta_{2}\left(\left(\frac{1}{M_{F}\left(\ell \xi_{2 j+2}, \ell \xi_{2 j+3}, t\right)}-1\right)+\left(\frac{1}{M_{F}\left(\ell \xi_{2 j+1}, \ell \xi_{2 j+2}, t\right)}-1\right)\right. \\
& \left.+\left(\frac{1}{M_{F}\left(\ell \xi_{2 j+1}, \ell \xi_{2 j+2}, t\right)}-1\right)+\left(\frac{1}{M_{F}\left(\ell \xi_{2 j+2}, \ell \xi_{2 j+3}, t\right)}-1\right)\right) \\
& +\beta_{3} \max \left\{\left(\frac{1}{M_{F}\left(\ell \xi_{2 j+2}, \ell \xi_{2 j+3}, t\right)}-1\right),\left(\frac{1}{M_{F}\left(\ell \xi_{2 j+1}, \ell \xi_{2 j+2}, t\right)}-1\right)\right. \\
& \left.+\left(\frac{1}{M_{F}\left(\ell \xi_{2 j+1}, \ell \xi_{2 j+3}, t\right)}-1\right)\right\} \cdot
\end{aligned}
$$

After simplification, for $t>0$, we obtain

$$
\begin{aligned}
& \frac{1}{M_{F}\left(\ell \xi_{2 j+2}, \ell \xi_{2 j+3}, t\right)}-1 \\
& \leq\left(\frac{\beta_{1}+2 \beta_{2}}{1-2 \beta_{2}}\right)\left(\frac{1}{M_{F}\left(\ell \xi_{2 j+1}, \ell \xi_{2 j+2}, t\right)}-1\right) \\
& \quad+\left(\frac{\beta_{3}}{1-2 \beta_{2}}\right) \max \left\{\left(\frac{1}{M_{F}\left(\ell \xi_{2 j+2}, \ell \xi_{2 j+3}, t\right)}-1\right),\right. \\
& \\
& \quad\left(\frac{1}{M_{F}\left(\ell \xi_{2 j+1}, \ell \xi_{2 j+2}, t\right)}-1\right), \\
& \\
& \left.\quad\left(\frac{1}{M_{F}\left(\ell \xi_{2 j+1}, \ell \xi_{2 j+3}, t\right)}-1\right)\right\} .
\end{aligned}
$$

Now three possibilities arise,

(i) If $\left(1 / M_{F}\left(\ell \xi_{2 j+2}, \ell \xi_{2 j+3}, t\right)-1\right)$ is a maximum term in (14) for $t>0$, then after simplification, we get that

$$
\begin{gathered}
\frac{1}{M_{F}\left(\ell \xi_{2 j+2}, \ell \xi_{2 j+3}, t\right)}-1 \leq\left(\frac{\beta_{1}+2 \beta_{2}}{1-2 \beta_{2}-\beta_{3}}\right) \\
\cdot\left(\frac{1}{M_{F}\left(\ell \xi_{2 j+1}, \ell \xi_{2 j+2}, t\right)}-1\right) \text { for } t>0 .
\end{gathered}
$$

(ii) If $\left(1 / M_{F}\left(\ell \xi_{2 j+1}, \ell \xi_{2 j+2}, t\right)-1\right)$ is a maximum term in (14) for $t>0$, then after simplification, we get that

$$
\begin{gathered}
\frac{1}{M_{F}\left(\ell \xi_{2 j+2}, \ell \xi_{2 j+3}, t\right)}-1 \leq\left(\frac{\beta_{1}+2 \beta_{2}+\beta_{3}}{1-2 \beta_{2}}\right) \\
\cdot\left(\frac{1}{M_{F}\left(\ell \xi_{2 j+1}, \ell \xi_{2 j+2}, t\right)}-1\right) \text { for } t>0 .
\end{gathered}
$$

(iii) If $\left(1 / M_{F}\left(\ell \xi_{2 j+1}, \ell \xi_{2 j+3}, t\right)-1\right)$ is a maximum term in (14) for $t>0$, then after simplification, we get that

$$
\begin{gathered}
\frac{1}{M_{F}\left(\ell \xi_{2 j+2}, \ell \xi_{2 j+3}, t\right)}-1 \leq\left(\frac{\beta_{1}+2 \beta_{2}+\beta_{3}}{1-2 \beta_{2}-\beta_{3}}\right) \\
\cdot\left(\frac{1}{M_{F}\left(\ell \xi_{2 j+1}, \ell \xi_{2 j+2}, t\right)}-1\right) \text { for } t>0 .
\end{gathered}
$$

Since $\lambda=\max \left\{\left(\beta_{1}+2 \beta_{2}+\beta_{3}\right) /\left(1-2 \beta_{2}\right),\left(\beta_{1}+2 \beta_{2}\right) /(1\right.$ $\left.\left.-2 \beta_{2}-\beta_{3}\right),\left(\beta_{1}+2 \beta_{2}+\beta_{3}\right) /\left(1-2 \beta_{2}-\beta_{3}\right)\right\}<1$, as defined in (12), then from (15), (16), and (17), we get that

$$
\frac{1}{M_{F}\left(\ell \xi_{2 j+2}, \ell \xi_{2 j+3}, t\right)}-1 \leq \lambda\left(\frac{1}{M_{F}\left(\ell \xi_{2 j+1}, \ell \xi_{2 j+2}, t\right)}-1\right) \text { for } t>0 \text {. }
$$



have

Now, from (12) and (18), and by induction, for $t>0$, we

$$
\begin{aligned}
& \frac{1}{M_{F}\left(\ell \xi_{2 j+2}, \ell \xi_{2 j+3}, t\right)}-1 \leq \lambda\left(\frac{1}{M_{F}\left(\ell \xi_{2 j+1}, \ell \xi_{2 j+2}, t\right)}-1\right) \\
& \quad \leq \lambda^{2}\left(\frac{1}{M_{F}\left(\ell \xi_{2 j}, \ell \xi_{2 j+1}, t\right)}-1\right) \\
& \quad \leq \cdots \leq \lambda^{2 j+2}\left(\frac{1}{M_{F}\left(\ell \xi_{0}, \ell \xi_{1}, t\right)}-1\right) \longrightarrow 0, \text { as } j \longrightarrow \infty
\end{aligned}
$$

Hence, from the above, we get that the sequence $\left\{\ell \xi_{j}\right\}_{j \geq 0}$ is a fuzzy-contractive in $\Omega$, therefore,

$$
\lim _{j \longrightarrow \infty} M_{F}\left(\ell \xi_{j}, \ell \xi_{j+1}, t\right)=1 \text { for } t>0 .
$$

Next, we have to prove that the sequence $\left\{\ell \xi_{j}\right\}_{j \geq 0}$ is a Cauchy sequence in $\left(\Omega, M_{F}, *\right)$. Then, from Definition 2 and by using (20), for $m>j$ where $m=j+n$ for $n=1,2, \cdots$ and $t>0$, we have that

$$
\begin{aligned}
& M_{F}\left(\ell \xi_{j}, \ell \xi_{m}, t\right)=M_{F}\left(\ell \xi_{j}, \ell \xi_{j+n}, t\right) \\
& \quad \geq M_{F}\left(\ell \xi_{j}, \ell \xi_{j+1}, \frac{t}{n}\right) * M_{F}\left(\ell \xi_{j+1}, \ell \xi_{j+2}, \frac{t}{n}\right) * \cdots * M_{F} \\
& \quad\left(\ell \xi_{j+n-1}, \ell \xi_{j+n}, \frac{t}{n}\right) \longrightarrow 1 * 1 * \cdots * 1=1, \text { as } j \longrightarrow \infty .
\end{aligned}
$$

This implies that

$$
\lim _{j, m \longrightarrow \infty} M_{F}\left(\ell \xi_{j}, \ell \xi_{m}, t\right)=1 \text { for } t>0 .
$$

Hence, proved that $\left\{\ell \xi_{j}\right\}$ is a Cauchy sequence. Since, $\ell(\Omega)$ is a complete subspace of $\left(\Omega, M_{F}, *\right)$, then there exits $\omega, \kappa \in \Omega$ such that $\ell \xi_{j} \longrightarrow \kappa=\ell \omega$ as $j \longrightarrow \infty$, therefore,

$$
\lim _{j \longrightarrow \infty} M_{F}\left(\ell \xi_{j}, \kappa, t\right)=\lim _{j \longrightarrow \infty} M_{F}\left(\ell \xi_{j}, \ell \omega, t\right)=1 \text { for } t>0 .
$$

Now, we have to show that $\ell \omega=G \omega=H \omega$. First, we estimate that $M_{F}(\ell \omega, G \omega, t)=M_{F}(\kappa, G \omega, t)$ for $t>0$. Since, $M_{F}$ is triangular, therefore,

$$
\begin{gathered}
\frac{1}{M_{F}(\ell \omega, G \omega, t)}-1 \leq\left(\frac{1}{M_{F}\left(\ell \omega, \ell \xi_{2 j+2}, t\right)}-1\right) \\
+\left(\frac{1}{M_{F}\left(\ell \xi_{2 j+2}, G \omega, t\right)}-1\right) \text { for } t>0 .
\end{gathered}
$$

Now by using (5), (20), and (23), for $t>0$, we have

$$
\begin{aligned}
& \frac{1}{M_{F}\left(\ell \xi_{2 j+2}, G \omega, t\right)}-1=\frac{1}{M_{F}\left(G \omega, H \xi_{2 j+1}, t\right)} \\
& \quad-1 \leq \beta_{1}\left(\frac{1}{M_{F}\left(\ell \omega, \ell \xi_{2 j+1}, t\right)}-1\right) \\
&+\beta_{2}\left(\left(\frac{1}{M_{F}(\ell \omega, G \omega, t)}-1\right)+\left(\frac{1}{M_{F}\left(\ell \xi_{2 j+1}, H \xi_{2 j+1}, t\right)}-1\right)\right. \\
&\left.+\left(\frac{1}{M_{F}\left(\ell \xi_{2 j+1}, G \omega, t\right)}-1\right)+\left(\frac{1}{M_{F}\left(\ell \omega, H \xi_{2 j+1}, t\right)}-1\right)\right) \\
&+\beta_{3} \max \left\{\left(\frac{1}{M_{F}(\ell \omega, G \omega, t)}-1\right),\left(\frac{1}{M_{F}\left(\ell \xi_{2 j+1}, H \xi_{2 j+1}, t\right)}-1\right),\right. \\
&\left.+\left(\frac{1}{M_{F}\left(\ell \xi_{2 j+1}, G \omega, t\right)}-1\right),\left(\frac{1}{M_{F}\left(\ell \omega, H \xi_{2 j+1}, t\right)}-1\right)\right\} \\
&=\beta_{1}\left(\frac{1}{M_{F}\left(\ell \omega, \ell \xi_{2 j+1}, t\right)}-1\right)+\beta_{2}\left(\left(\frac{1}{M_{F}(\ell \omega, G \omega, t)}-1\right)\right. \\
&+\left(\frac{1}{M_{F}\left(\ell \xi_{2 j+1}, \ell \xi_{2 j+2}, t\right)}-1\right)+\left(\frac{1}{M_{F}\left(\ell \xi_{2 j+1}, G \omega, t\right)}-1\right) \\
&\left.+\left(\frac{1}{M_{F}\left(\ell \omega, \ell \xi_{2 j+2}, t\right)}-1\right)\right)+\beta_{3} \max \left\{\left(\frac{1}{M_{F}(\ell \omega, G \omega, t)}-1\right),\right. \\
&\left(\frac{1}{M_{F}\left(\ell \xi_{2 j+1}, \ell \xi_{2 j+2}, t\right)}-1\right),\left(\frac{1}{M_{F}\left(\ell \xi_{2 j+1}, G \omega, t\right)}-1\right), \\
&\left.\left(\frac{1}{M_{F}\left(\ell \omega, \ell \xi_{2 j+2}, t\right)}-1\right)\right\} \\
& \longrightarrow\left(2 \beta_{2}+\beta_{3}\right)\left(\frac{1}{M_{F}(\ell \omega, G \omega, t)}-1\right) \text { as } \longrightarrow \infty .
\end{aligned}
$$

Thus,

$$
\begin{aligned}
& \lim _{j \longrightarrow \infty} \sup \left(\frac{1}{M_{F}\left(\ell \xi_{2 j+2}, G \omega, t\right)}-1\right) \\
& \quad \leq\left(2 \beta_{2}+\beta_{3}\right)\left(\frac{1}{M_{F}(\ell \omega, G \omega, t)}-1\right) \text { for } t>0 .
\end{aligned}
$$

The above (26) is together with (23) and (24), we get that

$$
\begin{aligned}
& \frac{1}{M_{F}(\ell \omega, G \omega, t)}-1 \\
& \quad \leq\left(2 \beta_{2}+\beta_{3}\right)\left(\frac{1}{M_{F}(\ell \omega, G \omega, t)}-1\right) \\
& \quad \Rightarrow\left(1-2 \beta_{2}-\beta_{3}\right)\left(\frac{1}{M_{F}(\ell \omega, G \omega, t)}-1\right) \leq 0 \text { for } t>0,
\end{aligned}
$$

which is a contradiction, as $\left(1-2 \beta_{2}-\beta_{3}\right) \neq 0$, therefore, $M_{F}(\ell \omega, G \omega, t)=1 \Rightarrow \ell \omega=G \omega$, for $t>0$. Hence, $\ell \omega=G \omega=\kappa$. 
Similarly, again by using the triangular property of $M_{F}$,

$$
\begin{gathered}
\frac{1}{M_{F}(\ell \omega, H \omega, t)}-1 \leq\left(\frac{1}{M_{F}\left(\ell \omega, \ell \xi_{2 j+1}, t\right)}-1\right) \\
+\left(\frac{1}{M_{F}\left(\ell \xi_{2 j+1}, H \omega, t\right)}-1\right) \text { for } t>0 .
\end{gathered}
$$

Again by using (5), (20), and (23), similar to the above simplification, we get

$$
\begin{aligned}
& \lim _{j \longrightarrow \infty} \sup \left(\frac{1}{M_{F}\left(\ell \xi_{2 j+1}, H \omega, t\right)}-1\right) \\
& \quad \leq\left(2 \beta_{2}+\beta_{3}\right)\left(\frac{1}{M_{F}(\ell \omega, H \omega, t)}-1\right) \text { for } t>0 .
\end{aligned}
$$

The above (29) is together with (23) and (28), we get that

$$
\begin{aligned}
& \frac{1}{M_{F}(\ell \omega, H \omega, t)}-1 \\
& \quad \leq\left(2 \beta_{2}+\beta_{3}\right)\left(\frac{1}{M_{F}(\ell \omega, H \omega, t)}-1\right) \\
& \quad \Rightarrow\left(1-2 \beta_{2}-\beta_{3}\right)\left(\frac{1}{M_{F}(\ell \omega, H \omega, t)}-1\right) \leq 0 \text { for } t>0,
\end{aligned}
$$

which is a contradiction, as $\left(1-2 \beta_{2}-\beta_{3}\right) \neq 0$, therefore, $M_{F}(\ell \omega, H \omega, t)=1 \Rightarrow \ell \omega=H \omega$, for $t>0$. Hence, $\ell \omega=H \omega=$ $\kappa$. It follows that $\omega$ is a coincidence point, and $\kappa$ is a common point of coincidence point for the self-mappings $\ell, G$, and $H$, that is,

$$
\ell \omega=G \omega=H \omega=\kappa .
$$

Next, we prove that the point of coincidence point of the three self-mappings is unique. Let $\exists$ another point $\kappa_{1} \in \Omega$ such that

$$
\kappa_{1}=\ell \omega_{1}=G \omega_{1}=H \omega_{1} \text { for some } \omega_{1} \in \Omega \text {. }
$$

Now by using (5), for $t>0$,

$$
\begin{aligned}
& \frac{1}{M_{F}\left(\kappa, \kappa_{1}, t\right)}-1=\left(\frac{1}{M_{F}\left(G \omega, H \omega_{1}, t\right)}-1\right) \\
& \quad \leq \beta_{1}\left(\frac{1}{M_{F}\left(\ell \omega, \ell \omega_{1}, t\right)}-1\right)+\beta_{2}\left(\left(\frac{1}{M_{F}(\ell \omega, G \omega, t)}-1\right)\right. \\
& \quad+\left(\frac{1}{M_{F}\left(\ell \omega_{1}, H \omega_{1}, t\right)}-1\right)+\left(\frac{1}{M_{F}\left(\ell \omega_{1}, G \omega, t\right)}-1\right) \\
& \left.\quad+\left(\frac{1}{M_{m}\left(\ell \omega, H \omega_{1}, t\right)}-1\right)\right)+\beta_{3} \max \left\{\left(\frac{1}{M_{F}(\ell \omega, G \omega, t)}-1\right)\right. \\
& \quad\left(\frac{1}{M_{F}\left(\ell \omega_{1}, H \omega_{1}, t\right)}-1\right),\left(\frac{1}{M_{F}\left(\ell \omega_{1}, G \omega, t\right)}-1\right), \\
& \left.\quad\left(\frac{1}{M_{m}\left(\ell \omega, H \omega_{1}, t\right)}-1\right)\right\}=\left(\beta_{1}+2 \beta_{2}+\beta_{3}\right)\left(\frac{1}{M_{F}\left(\kappa, \kappa_{1}, t\right)}-1\right) .
\end{aligned}
$$

After simplification, we get that

$$
\begin{aligned}
& \frac{1}{M_{F}\left(\kappa, \kappa_{1}, t\right)}-1 \\
& \quad \leq\left(\beta_{1}+2 \beta_{2}+\beta_{3}\right)\left(\frac{1}{M_{F}\left(\kappa, \kappa_{1}, t\right)}-1\right), \\
& \quad \Rightarrow\left(1-\beta_{1}-2 \beta_{2}-\beta_{3}\right)\left(\frac{1}{M_{F}\left(\kappa, \kappa_{1}, t\right)}-1\right) \leq 0 \text { for } t>0,
\end{aligned}
$$

which is a contradiction. Hence, $M_{F}\left(\kappa, \kappa_{1}, t\right)=1 \Rightarrow \kappa=\kappa_{1}$ for $t>0$. Further, by using the weak compatibility of the pairs $(G, \ell)$ and $(H, \ell)$ and by Proposition 10 , it follows that the three self-mappings $G, H$, and $\ell$ have a unique CFP, that is, $l \kappa=G \kappa=H \kappa=\kappa$.

Corollary 12. Let $\left(\Omega, M_{F}, *\right)$ be a FM space in which a FM $M_{F}$ is triangular. Let $G, H, \ell: \Omega \longrightarrow \Omega$ be three selfmappings that satisfy

$$
\begin{aligned}
& \frac{1}{M_{F}(G \xi, H \eta, t)}-1 \leq \beta_{1}\left(\frac{1}{M_{F}(\ell \xi, \ell \eta, t)}-1\right) \\
& +\beta_{2}\left(\left(\frac{1}{M_{F}(\ell \xi, G \xi, t)}-1\right)+\left(\frac{1}{M_{F}(\ell \eta, H \eta, t)}-1\right)\right. \\
& \left.+\left(\frac{1}{M_{F}(\ell \eta, G \xi, t)}-1\right)+\left(\frac{1}{M_{F}(\ell \xi, H \eta, t)}-1\right)\right),
\end{aligned}
$$

for all $\xi, \eta \in \Omega, t>0$, and $\beta_{1}, \beta_{2} \in[0,1)$ with $\left(\beta_{1}+4 \beta_{2}\right)<1$. If $G(\Omega) \cup H(\Omega) \subset \ell(\Omega)$ and $\ell(\Omega)$ is a complete subspace of $\Omega$. Then $G, H$, and $\ell$ have a unique point of coincidence. In addition, if $(G, \ell)$ and $(H, \ell)$ are weakly-compatible, then, $G, H$, and $\ell$ have a unique CFP.

Corollary 13. Let $\left(\Omega, M_{F}, *\right)$ be a FM space in which a FM $M_{F}$ is triangular. Let $G, H, \ell: \Omega \longrightarrow \Omega$ be three selfmappings that satisfy

$$
\begin{aligned}
& \frac{1}{M_{F}(G \xi, H \eta, t)}-1 \leq \beta_{1}\left(\frac{1}{M_{F}(\ell \xi, \ell \eta, t)}-1\right) \\
& +\beta_{3} \max \left\{\left(\frac{1}{M_{F}(\ell \xi, G \xi, t)}-1\right),\left(\frac{1}{M_{F}(\ell \eta, H \eta, t)}-1\right)\right. \\
& \left.\left(\frac{1}{M_{F}(\ell \xi, H \eta, t)}-1\right),\left(\frac{1}{M_{F}(\ell \eta, G \xi, t)}-1\right),\right\}
\end{aligned}
$$

for all $\xi, \eta \in \Omega, t>0$, and $\beta_{1}, \beta_{3} \in[0,1)$ with $\left(\beta_{1}+2 \beta_{3}\right)<1$. If $G(\Omega) \cup H(\Omega) \subset \ell(\Omega)$ and $\ell(\Omega)$ is a complete subspace of $\Omega$. Then, $G, H$, and $\ell$ have a unique point of coincidence. In addition, if $(G, \ell)$ and $(H, \ell)$ are weakly-compatible, then $G, H$, and $\ell$ have a unique CFP.

If we use identity map instead of the mapping $\ell$, i.e., $\ell=I$ , in Theorem 11, we get the following corollary. 
Corollary 14. Let $\left(\Omega, M_{F}, *\right)$ be a complete $F M$ space in which a $F M M_{F}$ is triangular. Let $G, H: \Omega \longrightarrow \Omega$ be a pair of self-mappings that satisfy

$$
\begin{aligned}
& \frac{1}{M_{F}(G \xi, H \eta, t)}-1 \leq \beta_{1}\left(\frac{1}{M_{F}(\xi, \eta, t)}-1\right) \\
& \quad+\beta_{2}\left(\left(\frac{1}{M_{F}(\xi, G \xi, t)}-1\right)+\left(\frac{1}{M_{F}(\eta, H \eta, t)}-1\right)\right. \\
& \left.\quad+\left(\frac{1}{M_{F}(\eta, G \xi, t)}-1\right)+\left(\frac{1}{M_{F}(\xi, H \eta, t)}-1\right)\right) \\
& \quad+\beta_{3} \max \left\{\left(\frac{1}{M_{F}(\xi, G \xi, t)}-1\right),\left(\frac{1}{M_{F}(\eta, H \eta, t)}-1\right),\right. \\
& \left.\left(\frac{1}{M_{F}(\xi, H \eta, t)}-1\right),\left(\frac{1}{M_{F}(\eta, G \xi, t)}-1\right),\right\},
\end{aligned}
$$

for all $\xi, \eta \in \Omega, t>0$, and $\beta_{1}, \beta_{2}, \beta_{3} \in[0,1)$ with $\left(\beta_{1}+4 \beta_{2}+\right.$ $\left.2 \beta_{3}\right)<1$. Then, the mappings $G$ and $H$ have a unique CFP.

Example 15. Let $\Omega=[0,1]$ and $*$ is a continuous $t$-norm. Let a FM $M_{F}: \Omega \times \Omega \times(0, \infty) \longrightarrow[0,1]$ be defined by

$M_{F}(\xi, \eta, t)=t / t+m(\xi, \eta)$, where $m(\xi, \eta)=|\xi-\eta|, \forall \xi, \eta \in \Omega$, and $t>0$.

Then easily one can verify that $M_{F}$ is triangular FM space $\left(\Omega, M_{F}, *\right)$. Now we define the mappings $G, H, \ell$ $: \Omega \longrightarrow \Omega$ by $G \xi=H \xi=2 \xi / 27$ and $\ell \xi=\xi / 3$ for all $\xi \in \Omega$ and satisfying the hypothesis $G(\Omega) \cup H(\Omega) \subset \ell(\Omega)$. By using the constants $\beta_{1}=2 / 9, \beta_{2}=1 / 6$, and $\beta_{3}=1 / 20$ in (5), then the three self-mappings satisfying the inequality (5) of Theorem 11. For verification, we present the following calculation for the defined mappings with constant values used in the inequality (5), for $t>0$, we have

$$
\begin{aligned}
& \frac{1}{M_{F}(G \xi, H \eta, t)}-1 \\
&=\frac{|G \xi-H \eta|}{t}=\frac{2|\xi-\eta|}{27 t} \leq \frac{13 \xi+\eta}{81 t} \leq \frac{2|\xi-\eta|}{27 t}+\frac{7(x+y)}{81 t} \\
&+\frac{1}{540 t} \max \{7 \xi, 7 \eta,|9 \eta-2 \xi|,|9 \xi-2 \eta|\} \\
& \leq \frac{2|\xi-\eta|}{27 t}+\frac{1}{6 t}\left(\frac{14(x+y)}{27}\right) \\
&+\frac{1}{20 t} \max \left\{\frac{7 \xi}{27}, \frac{7 \eta}{27}, \frac{|9 \eta-2 \xi|}{27}, \frac{|9 \xi-2 \eta|}{27}\right\} \\
& \leq \frac{2}{9 t}\left|\frac{\xi}{3}-\frac{\eta}{3}\right|+\frac{1}{6 t}\left(\left|\frac{\xi}{3}-\frac{2 \xi}{27}\right|+\left|\frac{\eta}{3}-\frac{2 \eta}{27}\right|+\left|\frac{\eta}{3}-\frac{2 \xi}{27}\right|+\left|\frac{\xi}{3}-\frac{2 \eta}{27}\right|\right) \\
&+\frac{1}{20 t} \max \left\{\left|\frac{\xi}{3}-\frac{2 \xi}{27}\right|,\left|\frac{\eta}{3}-\frac{2 \eta}{27}\right|,\left|\frac{\eta}{3}-\frac{2 \xi}{27}\right|,\left|\frac{\xi}{3}-\frac{2 \eta}{27}\right|\right\} \\
&= \beta_{1}\left(\frac{1}{M_{F}(\ell \xi, \ell \eta, t)}-1\right)+\beta_{2}\left(\left(\frac{1}{M_{F}(\ell \xi, G \xi, t)}-1\right)\right. \\
&+\left.\left(\frac{1}{M_{F}(\ell \eta, H \eta, t)}-1\right)+\left(\frac{1}{M_{F}(\ell \eta, G \xi, t)}-1\right)+\left(\frac{1}{M_{F}(\ell \xi, H \eta, t)}-1\right)\right) \\
&+ \beta_{3} \max \left\{\left(\frac{1}{M_{F}(\ell \xi, G \xi, t)}-1\right),\left(\frac{1}{M_{F}(\ell \eta, H \eta, t)}-1\right),\right. \\
&(\left.\left.\frac{1}{M_{F}(\ell \xi, H \eta, t)}-1\right),\left(\frac{1}{M_{F}(\ell \eta, G \xi, t)}-1\right)\right\} .
\end{aligned}
$$

Hence, proved that all the conditions of Theorem 11 are satisfied with $\beta_{1}=2 / 9, \beta_{2}=1 / 6$, and $\beta_{3}=1 / 20$, where $\beta_{1}+4 \beta_{2}+2 \beta_{3}=(2 / 9+4(1 / 6)+2(1 / 20))=89 / 90<1 \quad$ and the three-self-mappings $G, H$, and $\ell$ have a unique coincidence point and CFP, that is, $0 \in \Omega$.

Theorem 16. Let $\left(\Omega, M_{F}, *\right)$ be a FM space in which a FM $M_{F}$ is triangular. Let $G, H, \ell: \Omega \longrightarrow \Omega$ be three selfmappings that satisfy

$$
\begin{aligned}
& \frac{1}{M_{F}(G \xi, H \eta, t)}-1 \leq \beta_{1}\left(\frac{1}{M_{F}(\ell \xi, \ell \eta, t)}-1\right) \\
& +\beta_{2}\left(\frac{1}{M_{F}(\ell \xi, G \xi, t)}-1\right)+\beta_{3}\left(\frac{1}{M_{F}(\ell \eta, H \eta, t)}-1\right) \\
& +\beta_{4}\left(\frac{1}{M_{F}(\ell \eta, G \xi, t)}-1\right)+\beta_{5}\left(\frac{1}{M_{F}(\ell \xi, H \eta, t)}-1\right) \\
& +\beta_{6}\left(\frac{1}{\aleph(G, H, \ell, \xi, \eta, t)}-1\right),
\end{aligned}
$$

where

$$
\begin{gathered}
\frac{1}{\aleph(G, H, \ell, \xi, \eta, t)}-1=\min \left\{\left(\frac{1}{M_{F}(\ell \xi, G \xi, t)}-1\right),\right. \\
\left(\frac{1}{M_{F}(\ell \eta, H \eta, t)}-1\right),\left(\frac{1}{M_{F}(\ell \xi, H \eta, t)}-1\right), \\
\left.\left(\frac{1}{M_{F}(\ell \eta, G \xi, t)}-1\right),\right\},
\end{gathered}
$$

for all $\xi, \eta \in \Omega, t>0$, and $\beta_{1}, \beta_{2}, \beta_{3}, \beta_{4}, \beta_{5}, \beta_{6} \in[0,1)$ with ( $\left.\beta_{1}+\beta_{2}+\beta_{3}+\beta_{4}+\beta_{5}+\beta_{6}\right)<1$ and $\beta_{2}=\beta_{3}$ or $\beta_{4}=\beta_{5}$. If $G$ $(\Omega) \cup H(\Omega) \subset \ell(\Omega)$ and $\ell(\Omega)$ is a complete subspace of $\Omega$. Then $G, H$, and $\ell$ have a unique point of coincidence. In addition, if $(G, \ell)$ and $(H, \ell)$ are weakly-compatible, then $G, H$, and $\ell$ have a unique CFP.

Proof. Let $\xi_{0} \in \Omega$ be arbitrary and by using the hypothesis $G$ ( $\Omega) \cup H(\Omega) \subset \ell(\Omega)$. Now, we choose a sequence $\left\{\xi_{j}\right\}$ such that

$$
\ell \xi_{2 j+1}=G \xi_{2 j} \text { and } \ell \xi_{2 j+2}=H \xi_{2 j+1} \text { for } j \geq 0
$$

Now, from (40), for $t>0$, we have

$$
\begin{aligned}
& \frac{1}{M_{F}\left(\ell \xi_{2 j+1}, \ell \xi_{2 j+2}, t\right)}-1 \\
& =\frac{1}{M_{F}\left(G \xi_{2 j}, H \xi_{2 j+1}, t\right)}-1 \leq \beta_{1}\left(\frac{1}{M_{F}\left(\ell \xi_{2 j}, \ell \xi_{2 j+1}, t\right)}-1\right) \\
& \quad+\beta_{2}\left(\frac{1}{M_{F}\left(\ell \xi_{2 j}, G \xi_{2 j}, t\right)}-1\right)+\beta_{3}\left(\frac{1}{M_{F}\left(\ell \xi_{2 j+1}, H \xi_{2 j+1}, t\right)}-1\right)
\end{aligned}
$$




$$
\begin{aligned}
& +\beta_{4}\left(\frac{1}{M_{F}\left(\ell \xi_{2 j+1}, G \xi_{2 j}, t\right)}-1\right) \\
& +\beta_{5}\left(\frac{1}{M_{F}\left(\ell \xi_{2 j}, H \xi_{2 j+1}, t\right)}-1\right) \\
& +\beta_{6}\left(\frac{1}{\aleph\left(G, H, \ell, \xi_{2 j}, \xi_{2 j+1}, t\right)}-1\right) \\
& =\beta_{1}\left(\frac{1}{M_{F}\left(\ell \xi_{2 j}, \ell \xi_{2 j+1}, t\right)}-1\right) \\
& +\beta_{2}\left(\frac{1}{M_{F}\left(\ell \xi_{2 j}, \ell \xi_{2 j+1}, t\right)}-1\right) \\
& +\beta_{3}\left(\frac{1}{M_{F}\left(\ell \xi_{2 j+1}, \ell \xi_{2 j+2}, t\right)}-1\right) \\
& +\beta_{5}\left(\frac{1}{M_{F}\left(\ell \xi_{2 j}, \ell \xi_{2 j+2}, t\right)}-1\right) \\
& +\beta_{6}\left(\frac{1}{\aleph\left(G, H, \ell, \xi_{2 j}, \xi_{2 j+1}, t\right)}-1\right) \\
& \leq\left(\beta_{1}+\beta_{2}+\beta_{5}\right)\left(\frac{1}{M_{F}\left(\ell \xi_{2 j}, \ell \xi_{2 j+1}, t\right)}-1\right) \\
& +\left(\beta_{3}+\beta_{5}\right)\left(\frac{1}{M\left(\ell \xi_{2 j+1}, \ell \xi_{2 j+2}, t\right)}-1\right) \\
& +
\end{aligned}
$$

where

$$
\begin{aligned}
& \frac{1}{\aleph\left(G, H, \ell, \xi_{2 j}, \xi_{2 j+1}, t\right)}-1 \\
& =\min \left\{\left(\frac{1}{M_{F}\left(\ell \xi_{2 j}, G \xi_{2 j}, t\right)}-1\right),\left(\frac{1}{M_{F}\left(\ell \xi_{2 j+1}, H \xi_{2 j+1}, t\right)}-1\right),\right. \\
& \left.\left(\frac{1}{M_{F}\left(\ell \xi_{2 j+1}, G \xi_{2 j}, t\right)}-1\right),\left(\frac{1}{M_{F}\left(\ell \xi_{2 j}, H \xi_{2 j+1}, t\right)}-1\right)\right\} \\
& =\min \left\{\left(\frac{1}{M_{F}\left(\ell \xi_{2 j}, \ell \xi_{2 j+1}, t\right)}-1\right),\left(\frac{1}{M_{F}\left(\ell \xi_{2 j+1}, \ell \xi_{2 j+2}, t\right)}-1\right)\right. \text {, } \\
& \left.0,\left(\frac{1}{M_{F}\left(\ell \xi_{2 j}, \ell \xi_{2 j+2}, t\right)}-1\right)\right\}=0 \text {, }
\end{aligned}
$$

Hence, we get that

$\frac{1}{M_{F}\left(\ell \xi_{2 j+1}, \ell \xi_{2 j+2}, t\right)}-1 \leq \mathscr{A}\left(\frac{1}{M_{F}\left(\ell \xi_{2 j}, \ell \xi_{2 j+1}, t\right)}-1\right)$ for $t>0$,

where $\mathscr{A}=\left(\beta_{1}+\beta_{2}+\beta_{5}\right) /\left(1-\beta_{3}-\beta_{5}\right)$. Similarly, again by the view of (40), for $t>0$,

$$
\begin{aligned}
& \frac{1}{M_{F}\left(\ell \xi_{2 j+2}, \ell \xi_{2 j+3}, t\right)}-1=\frac{1}{M_{F}\left(G \xi_{2 j+2}, H \xi_{2 j+1}, t\right)}-1 \\
\leq & \beta_{1}\left(\frac{1}{M_{F}\left(\ell \xi_{2 j+2}, \ell \xi_{2 j+1}, t\right)}-1\right)+\beta_{2}\left(\frac{1}{M_{F}\left(\ell \xi_{2 j+2}, G \xi_{2 j+2}, t\right)}-1\right) \\
& +\beta_{3}\left(\frac{1}{M_{F}\left(\ell \xi_{2 j+1}, H \xi_{2 j+1}, t\right)}-1\right)+\beta_{4}\left(\frac{1}{M_{F}\left(\ell \xi_{2 j+1}, G \xi_{2 j+2}, t\right)}-1\right) \\
& +\beta_{5}\left(\frac{1}{M_{F}\left(\ell \xi_{2 j+2}, H \xi_{2 j+1}, t\right)}-1\right)+\beta_{6}\left(\frac{1}{\aleph\left(G, H, \ell, \xi_{2 j+2}, \xi_{2 j+1}, t\right)}-1\right) \\
= & \beta_{1}\left(\frac{1}{M_{F}\left(\ell \xi_{2 j+2}, \ell \xi_{2 j+1}, t\right)}-1\right)+\beta_{2}\left(\frac{1}{M_{F}\left(\ell \xi_{2 j+2}, \ell \xi_{2 j+3}, t\right)}-1\right) \\
& +\beta_{3}\left(\frac{1}{M_{F}\left(\ell \xi_{2 j+1}, \ell \xi_{2 j+2}, t\right)}-1\right)+\beta_{4}\left(\frac{1}{M_{F}\left(\ell \xi_{2 j+1}, \ell \xi_{2 j+3}, t\right)}-1\right) \\
& +\beta_{6}\left(\frac{1}{\aleph\left(G, H, \ell, \xi_{2 j+2}, \xi_{2 j+1}, t\right)}-1\right) \\
\leq & \left.\beta_{1}+\beta_{3}+\beta_{4}\right)\left(\frac{1}{M_{F}\left(\ell \xi_{2 j}, \ell \xi_{2 j+1}, t\right)}-1\right) \\
& +\left(\beta_{2}+\beta_{4}\right)\left(\frac{1}{M\left(\ell\left(\ell \xi_{2 j+1}, \ell \xi_{2 j+2}, t\right)\right.}-1\right) \\
& +\beta_{6}\left(\frac{1}{\aleph\left(G, H, \ell, \xi_{2 j+2}, \xi_{2 j+1}, t\right)}-1\right),
\end{aligned}
$$

where

$$
\begin{aligned}
& \frac{1}{\aleph\left(G, H, \ell, \xi_{2 j+2}, \xi_{2 j+1}, t\right)}-1 \\
& =\min \left\{\left(\frac{1}{M_{F}\left(l \xi_{2 j+2}, G \xi_{2 j+2}, t\right)}-1\right),\left(\frac{1}{M_{F}\left(l \xi_{2 j+1}, H \xi_{2 j+1}, t\right)}-1\right),\right. \\
& \left.\left(\frac{1}{M_{F}\left(\ell \xi_{2 j+1}, G \xi_{2 j+2}, t\right)}-1\right),\left(\frac{1}{M_{F}\left(\ell \xi_{2 j+2}, H \xi_{2 j+1}, t\right)}-1\right)\right\} \\
& =\min \left\{\left(\frac{1}{M_{F}\left(\ell \xi_{2 j+2}, \ell \xi_{2 j+3}, t\right)}-1\right),\left(\frac{1}{M_{F}\left(\ell \xi_{2 j+1}, \ell \xi_{2 j+2}, t\right)}-1\right),\right. \\
& \left.\left(\frac{1}{M_{F}\left(\ell \xi_{2 j+1}, \ell \xi_{2 j+3}, t\right)}-1\right), 0\right\}=0 \text {. }
\end{aligned}
$$

Hence, we get that

$$
\frac{1}{M_{F}\left(\ell \xi_{2 j+2}, \ell \xi_{2 j+3}, t\right)}-1 \leq \mathscr{B}\left(\frac{1}{M_{F}\left(\ell \xi_{2 j+1}, \ell \xi_{2 j+2}, t\right)}-1\right) \text { for } t>0,
$$

where $\mathscr{B}=\left(\beta_{1}+\beta_{3}+\beta_{4}\right) /\left(1-\beta_{2}-\beta_{4}\right)$. Now from (45) and (48),

$$
\frac{1}{M_{F}\left(\ell \xi_{2 j+2}, \ell \xi_{2 j+3}, t\right)}-1 \leq \mathscr{B} \cdot \mathscr{A}\left(\frac{1}{M_{F}\left(\ell \xi_{2 j}, \ell \xi_{2 j+1}, t\right)}-1\right) \text { for } t>0 .
$$

Now by the view of (45), (48), (49), and by induction, for 
$t>0$, we obtain that

$$
\begin{aligned}
& \frac{1}{M_{F}\left(\ell \xi_{2 j+1}, \ell \xi_{2 j+2}, t\right)}-1 \\
& \quad \leq \mathscr{A}\left(\frac{1}{M_{F}\left(\ell \xi_{2 j}, \ell \xi_{2 j+1}, t\right)}-1\right) \\
& \quad \leq \mathscr{A} \cdot \mathscr{B}\left(\frac{1}{M_{F}\left(\ell \xi_{2 j-1}, \ell \xi_{2 j}, t\right)}-1\right) \\
& \leq \mathscr{A} \cdot \mathscr{B} \cdot \mathscr{A}\left(\frac{1}{M_{F}\left(\ell \xi_{2 j-2}, \ell \xi_{2 j-1}, t\right)}-1\right) \\
& \leq \ldots \leq \mathscr{A}(\mathscr{B} \cdot \mathscr{A})^{j}\left(\frac{1}{M_{F}\left(\ell \xi_{0}, \ell \xi_{1}, t\right)}-1\right) \\
& \frac{1}{M\left(\ell \xi_{2 j+2}, \ell \xi_{2 j+3}, t\right)}-1 \leq \mathscr{B}\left(\frac{1}{M\left(\ell \xi_{2 j+1}, \ell \xi_{2 j+2}, t\right)}-1\right) \\
& \leq \cdots \leq(\mathscr{B} \cdot \mathscr{A}){ }^{j+1}\left(\frac{1}{M\left(\ell \xi_{0}, \ell \xi_{1}, t\right)}-1\right)
\end{aligned}
$$

Since, $\mathscr{A}=\left(\beta_{1}+\beta_{2}+\beta_{5}\right) /\left(1-\beta_{3}-\beta_{5}\right)$ and $\mathscr{B}=\left(\beta_{1}+\beta_{3}\right.$ $\left.+\beta_{4}\right) /\left(1-\beta_{2}-\beta_{4}\right)$. Now, if $\beta_{2}=\beta_{3}$,

$$
\begin{aligned}
\mathscr{A} \cdot \mathscr{B}= & \frac{\beta_{1}+\beta_{2}+\beta_{5}}{1-\beta_{3}-\beta_{5}} \cdot \frac{\beta_{1}+\beta_{3}+\beta_{4}}{1-\beta_{2}-\beta_{4}} \\
& =\frac{\beta_{1}+\beta_{2}+\beta_{5}}{1-\beta_{2}-\beta_{4}} \cdot \frac{\beta_{1}+\beta_{3}+\beta_{4}}{1-\beta_{3}-\beta_{5}}<1 \cdot 1=1,
\end{aligned}
$$

In second case, if $\beta_{4}=\beta_{5}$,

$$
\mathscr{A} \cdot \mathscr{B}=\frac{\beta_{1}+\beta_{2}+\beta_{5}}{1-\beta_{3}-\beta_{5}} \cdot \frac{\beta_{1}+\beta_{3}+\beta_{4}}{1-\beta_{2}-\beta_{4}}<1 \cdot 1=1 .
$$

Hence, from (50), (52), and (53), we get that $\left\{\ell \xi_{j}\right\}_{j \geq 0}$ is a fuzzy-contractive sequence, therefore,

$$
\lim _{j \longrightarrow \infty} M_{F}\left(\ell \xi_{j}, \ell \xi_{j+1}, t\right)=1 \text { for } t>0 .
$$

Next, we have to prove that a sequence $\left\{\ell \xi_{j}\right\}_{j \geq 0}$ is a Cauchy sequence in $\left(\Omega, M_{F}, *\right)$. Then, from Definition 2 and by using (54), for $m>j$ where $m=j+n$ for $n=1,2, \cdots$ and $t>0$, we have that

$$
\begin{aligned}
M_{F}\left(\ell \xi_{j}, \ell \xi_{m}, t\right)= & M_{F}\left(\ell \xi_{j}, \ell \xi_{j+n}, t\right) \\
\geq & M_{F}\left(\ell \xi_{j}, \ell \xi_{j+1}, \frac{t}{n}\right) * M_{F}\left(\ell \xi_{j+1}, \ell \xi_{j+2}, \frac{t}{n}\right) \\
& * \cdots * M_{F}\left(\ell \xi_{j+n-1}, \ell \xi_{j+n}, \frac{t}{n}\right) \\
& \longrightarrow 1 * 1 * \cdots * 1=1, \text { as } j \longrightarrow \infty .
\end{aligned}
$$

This implies that

$$
\lim _{j, m \rightarrow \infty} M_{F}\left(\ell \xi_{j}, \ell \xi_{m}, t\right)=1 \text { for } t>0 .
$$

Hence, proved that $\left\{\ell \xi_{j}\right\}$ is a Cauchy sequence. Since, $\ell(\Omega)$ is a complete subspace of $\left(\Omega, M_{F}, *\right)$, then there exits $\omega, \kappa \in \Omega$ such that $\ell \xi_{j} \longrightarrow \kappa=\ell \omega$ as $j \longrightarrow \infty$, therefore,

$$
\lim _{j \longrightarrow \infty} M_{F}\left(\ell \xi_{j}, \kappa, t\right)=\lim _{j \longrightarrow \infty} M_{F}\left(\ell \xi_{j}, \ell \omega, t\right)=1 \text { for } t>0 .
$$

Now we have to show that $\ell \omega=G \omega=H \omega$. First, we estimate that $M_{F}(\ell \omega, G \omega, t)=M_{F}(\kappa, G \omega, t)$ for $t>0$. Since, $M_{F}$ is triangular, therefore,

$$
\begin{gathered}
\frac{1}{M_{F}(\ell \omega, G \omega, t)}-1 \leq\left(\frac{1}{M_{F}\left(\ell \omega, \ell \xi_{2 j+2}, t\right)}-1\right) \\
+\left(\frac{1}{M_{F}\left(\ell \xi_{2 j+2}, G \omega, t\right)}-1\right) \text { for } t>0 .
\end{gathered}
$$

Now by using (40), (54), and (57), for $t>0$, we have

$$
\begin{aligned}
& \frac{1}{M_{F}\left(\ell \xi_{2 j+2}, G \omega, t\right)}-1=\frac{1}{M_{F}\left(G \omega, H \xi_{2 j+1}, t\right)} \\
& -1 \leq \beta_{1}\left(\frac{1}{M_{F}\left(\ell \omega, \ell \xi_{2 j+1}, t\right)}-1\right) \\
& +\beta_{2}\left(\frac{1}{M_{F}(\ell \omega, G \omega, t)}-1\right) \\
& +\beta_{3}\left(\frac{1}{M_{F}\left(\ell \xi_{2 j+1}, H \xi_{2 j+1}, t\right)}-1\right) \\
& +\beta_{4}\left(\frac{1}{M_{F}\left(\ell \xi_{2 j+1}, G \omega, t\right)}-1\right) \\
& +\beta_{5}\left(\frac{1}{M_{F}\left(\ell \omega, H \xi_{2 j+1}, t\right)}-1\right) \\
& +\beta_{6}\left(\frac{1}{\aleph\left(G, H, \ell, \omega, \xi_{2 j+1}, t\right)}-1\right) \\
& =\beta_{1}\left(\frac{1}{M_{F}\left(\ell \omega, \ell \xi_{2 j+1}, t\right)}-1\right)+\beta_{2}\left(\frac{1}{M_{F}(\ell \omega, G \omega, t)}-1\right) \\
& +\beta_{3}\left(\frac{1}{M_{F}\left(\ell \xi_{2 j+1}, \ell \xi_{2 j+2}, t\right)}-1\right)+\beta_{4}\left(\frac{1}{M_{F}\left(\ell \xi_{2 j+1}, G \omega, t\right)}-1\right) \\
& +\beta_{5}\left(\frac{1}{M_{F}\left(\ell \omega, \ell \xi_{2 j+2}, t\right)}-1\right)+\beta_{6}\left(\frac{1}{\aleph\left(G, H, \ell, \omega, \xi_{2 j+1}, t\right)}-1\right) \\
& \longrightarrow\left(\beta_{2}+\beta_{4}\right)\left(\frac{1}{M_{F}(\ell \omega, G \omega, t)}-1\right) \\
& +\beta_{6}\left(\frac{1}{\aleph\left(G, H, \ell, \omega, \xi_{2 j+1}, t\right)}-1\right) \text {, as } j \longrightarrow \infty \text {, }
\end{aligned}
$$


where

$$
\begin{aligned}
\frac{1}{\aleph\left(G, H, \ell, \omega, \xi_{2 j+1}, t\right)} & -1=\min \left\{\left(\frac{1}{M_{F}(\ell \omega, G \omega, t)}-1\right),\left(\frac{1}{M_{F}\left(\ell \xi_{2 j+1}, H \xi_{2 j+1}, t\right)}-1\right),\right. \\
& \left.\left(\frac{1}{M_{F}\left(\ell \omega, H \xi_{2 j+1}, t\right)}-1\right),\left(\frac{1}{M_{F}\left(\ell \xi_{2 j+1}, G \omega, t\right)}-1\right)\right\} \\
= & \min \left\{\left(\frac{1}{M_{F}(\ell \omega, G \omega, t)}-1\right),\left(\frac{1}{M_{F}\left(\ell \xi_{2 j+1}, \ell \xi_{2 j+2}, t\right)}-1\right),\right. \\
& \left.\left(\frac{1}{M_{F}\left(\ell \omega, \ell \xi_{2 j+2}, t\right)}-1\right),\left(\frac{1}{M_{F}\left(\ell \xi_{2 j+1}, G \omega, t\right)}-1\right)\right\} \\
& \longrightarrow \min \left\{0,\left(\frac{1}{M_{F}(\ell \omega, G \omega, t)}-1\right)\right\}=0, \text { as } j \longrightarrow \infty .
\end{aligned}
$$

Thus,

$$
\begin{gathered}
\lim _{j \longrightarrow \infty} \sup \left(\frac{1}{M_{F}\left(\ell \xi_{2 j+2}, G \omega, t\right)}-1\right) \leq\left(\beta_{2}+\beta_{4}\right) \\
\cdot\left(\frac{1}{M_{F}(\ell \omega, G \omega, t)}-1\right) \text { for } t>0 .
\end{gathered}
$$

The above (61) is together with (57) and (58), we get that

$$
\begin{gathered}
\frac{1}{M_{F}(\ell \omega, G \omega, t)}-1 \leq\left(\beta_{2}+\beta_{4}\right)\left(\frac{1}{M_{F}(\ell \omega, G \omega, t)}-1\right) \\
\Rightarrow\left(1-\beta_{2}-\beta_{4}\right)\left(\frac{1}{M_{F}(\ell \omega, G \omega, t)}-1\right) \leq 0 \text { for } t>0,
\end{gathered}
$$

which is a contradiction, as $\left(1-\beta_{2}-\beta_{4}\right) \neq 0$, therefore, $M_{F}(\ell \omega, G \omega, t)=1 \Rightarrow \ell \omega=G \omega$, for $t>0$. Hence, $\ell \omega=G \omega=\kappa$.

Similarly, again by using the triangular property of $M_{F}$,

$$
\begin{gathered}
\frac{1}{M_{F}(\ell \omega, H \omega, t)}-1 \leq\left(\frac{1}{M_{F}\left(\ell \omega, \ell \xi_{2 j+1}, t\right)}-1\right) \\
+\left(\frac{1}{M_{F}\left(\ell \xi_{2 j+1}, H \omega, t\right)}-1\right) \text { for } t>0 .
\end{gathered}
$$

Again by using (40), (54), and (57), similar as above simplification, we get

$$
\begin{gathered}
\lim _{j \longrightarrow \infty} \sup \left(\frac{1}{M_{F}\left(\ell \xi_{2 j+1}, H \omega, t\right)}-1\right) \leq\left(\beta_{3}+\beta_{5}\right) \\
\left(\frac{1}{M_{F}(\ell \omega, H \omega, t)}-1\right) \text { for } t>0 .
\end{gathered}
$$

The above (64) is together with (57) and (63), we get that

$$
\begin{gathered}
\frac{1}{M_{F}(\ell \omega, H \omega, t)}-1 \leq\left(\beta_{3}+\beta_{5}\right)\left(\frac{1}{M_{F}(\ell \omega, H \omega, t)}-1\right) \\
\Rightarrow\left(1-\beta_{3}-\beta_{5}\right)\left(\frac{1}{M_{F}(\ell \omega, H \omega, t)}-1\right) \leq 0 \text { for } t>0,
\end{gathered}
$$

which is a contradiction, as $\left(1-\beta_{3}-\beta_{5}\right) \neq 0$, therefore, $M_{F}$ $(\ell \omega, H \omega, t)=1 \Rightarrow \ell \omega=H \omega$, for $t>0$. Hence, $\ell \omega=H \omega=\kappa$. It follows that $\omega$ is a coincidence point and $\kappa$ is a common point of coincidence point for the self-mappings $\ell, G$, and $H$, that is,

$$
\ell \omega=G \omega=H \omega=\kappa .
$$

Next, we prove that the point of coincidence point of the three self-mappings is unique. Let $\exists$ another point $\kappa_{1} \in \Omega$ such that

$$
\kappa_{1}=\ell \omega_{1}=G \omega_{1}=H \omega_{1} \text { for some } \omega_{1} \in \Omega .
$$

Now by using (40), for $t>0$,

$$
\begin{aligned}
& \frac{1}{M_{F}\left(\kappa, \kappa_{1}, t\right)}-1=\left(\frac{1}{M_{F}\left(G \omega, H \omega_{1}, t\right)}-1\right) \\
& \leq \beta_{1}\left(\frac{1}{M_{F}\left(\ell \omega, \ell \omega_{1}, t\right)}-1\right)+\beta_{2}\left(\frac{1}{M_{F}(\ell \omega, G \omega, t)}-1\right) \\
& \quad+\beta_{3}\left(\frac{1}{M_{F}\left(\ell \omega_{1}, H \omega_{1}, t\right)}-1\right)+\beta_{4}\left(\frac{1}{M_{F}\left(\ell \omega_{1}, G \omega, t\right)}-1\right) \\
& \quad+\beta_{5}\left(\frac{1}{M_{F}\left(\ell \omega, H \omega_{1}, t\right)}-1\right)+\beta_{6}\left(\frac{1}{\mathcal{N}\left(G, H, \ell, \omega, \omega_{1}, t\right)}-1\right) \\
& =\left(\beta_{1}+\beta_{4}+\beta_{5}\right)\left(\frac{1}{M_{F}\left(\kappa, \kappa_{1}, t\right)}-1\right)+\beta_{6}\left(\frac{1}{\mathcal{N}\left(G, H, \ell, \omega, \omega_{1}, t\right)}-1\right),
\end{aligned}
$$

where

$$
\begin{aligned}
& \frac{1}{\aleph\left(G, H, \ell, \omega, \omega_{1}, t\right)}-1=\min \left\{\left(\frac{1}{M_{F}(\ell \omega, G \omega, t)}-1\right),\right. \\
& \quad\left(\frac{1}{M_{F}\left(\ell \omega_{1}, H \omega_{1}, t\right)}-1\right),\left(\frac{1}{M_{F}\left(\ell \omega, H \omega_{1}, t\right)}-1\right), \\
& \left.\left(\frac{1}{M_{F}\left(\ell \omega_{1}, G \omega, t\right)}-1\right)\right\}=\min \left\{\left(\frac{1}{M_{F}\left(\kappa, \kappa_{1}, t\right)}-1\right), 0\right\}=0 .
\end{aligned}
$$

Hence, from the above, we get that

$$
\begin{aligned}
& \frac{1}{M_{F}\left(\kappa, \kappa_{1}, t\right)}-1 \leq\left(\beta_{1}+\beta_{4}+\beta_{5}\right)\left(\frac{1}{M_{F}\left(\kappa, \kappa_{1}, t\right)}-1\right), \\
& \quad \Longrightarrow\left(1-\beta_{1}-\beta_{4}-\beta_{5}\right)\left(\frac{1}{M_{F}\left(\kappa, \kappa_{1}, t\right)}-1\right) \leq 0 \text { for } t>0,
\end{aligned}
$$

which is a contradiction. Hence $M_{F}\left(\kappa, \kappa_{1}, t\right)=1 \Rightarrow \kappa=\kappa_{1}$ for $t>0$. Further, by using the weak compatibility of the pairs 
$(G, \ell)$ and $(H, \ell)$ and by Proposition 10, it follows that the three self-mappings $G, H$, and $\ell$ have a unique CFP, that is, $\ell \kappa=G \kappa=H \kappa=\kappa$.

Corollary 17. Let $\left(\Omega, M_{F}, *\right)$ be a FM space in which a FM $M_{F}$ is triangular. Let $G, H, \ell: \Omega \longrightarrow \Omega$ be three selfmappings that satisfy

$$
\begin{aligned}
& \frac{1}{M_{F}(G \xi, H \eta, t)}-1 \leq \beta_{1}\left(\frac{1}{M_{F}(\ell \xi, \ell \eta, t)}-1\right) \\
& +\beta_{2}\left(\frac{1}{M_{F}(\ell \xi, G \xi, t)}-1\right)+\beta_{3}\left(\frac{1}{M_{F}(\ell \eta, H \eta, t)}-1\right) \\
& \quad+\beta_{6}\left(\frac{1}{\aleph(G, H, \ell, \xi, \eta, t)}-1\right),
\end{aligned}
$$

where

$$
\begin{aligned}
& \frac{1}{\aleph(G, H, \ell, \xi, \eta, t)}-1=\min \left\{\left(\frac{1}{M_{F}(\ell \xi, G \xi, t)}-1\right),\right. \\
& \left(\frac{1}{M_{F}(\ell \eta, H \eta, t)}-1\right),\left(\frac{1}{M_{F}(\ell \xi, H \eta, t)}-1\right), \\
& \left.\left(\frac{1}{M_{F}(\ell \eta, G \xi, t)}-1\right),\right\},
\end{aligned}
$$

for all $\xi, \eta \in \Omega, t>0$, and $\beta_{1}, \beta_{2}, \beta_{3}, \beta_{6} \in[0,1)$ with $\left(\beta_{1}\right.$ $\left.+\beta_{2}+\beta_{3}\right)<1$. If $G(\Omega) \cup H(\Omega) \subset \ell(\Omega)$ and $\ell(\Omega)$ is a complete subspace of $\Omega$. Then $G, H$, and $\ell$ have a unique point of coincidence. In addition, if $(G, \ell)$ and $(H, \ell)$ are weaklycompatible, then $G, H$, and $\ell$ have a unique CFP.

Corollary 18. Let $\left(\Omega, M_{F}, *\right)$ be a FM space in which a FM $M_{F}$ is triangular. Let $G, H, \ell: \Omega \longrightarrow \Omega$ be three selfmappings that satisfy

$$
\begin{aligned}
& \frac{1}{M_{F}(G \xi, H \eta, t)}-1 \leq \beta_{1}\left(\frac{1}{M_{F}(\ell \xi, \ell \eta, t)}-1\right) \\
& \quad+\beta_{4}\left(\frac{1}{M_{F}(\ell \eta, G \xi, t)}-1\right)+\beta_{5}\left(\frac{1}{M_{F}(\ell \xi, H \eta, t)}-1\right) \\
& \quad+\beta_{6}\left(\frac{1}{\aleph(G, H, \ell, \xi, \eta, t)}-1\right),
\end{aligned}
$$

where

$$
\begin{aligned}
& \frac{1}{\aleph(G, H, \ell, \xi, \eta, t)}-1=\min \left\{\left(\frac{1}{M_{F}(\ell \xi, G \xi, t)}-1\right),\right. \\
& \left(\frac{1}{M_{F}(\ell \eta, H \eta, t)}-1\right),\left(\frac{1}{M_{F}(\ell \xi, H \eta, t)}-1\right), \\
& \left.\left(\frac{1}{M_{F}(\ell \eta, G \xi, t)}-1\right)\right\}
\end{aligned}
$$

for all $\xi, \eta \in \Omega, t>0$, and $\beta_{1}, \beta_{4}, \beta_{5}, \beta_{6} \in[0,1)$ with $\left(\beta_{1}+\beta_{4}\right.$ $\left.+\beta_{5}\right)<1$. If $G(\Omega) \cup H(\Omega) \subset \ell(\Omega)$ and $\ell(\Omega)$ is a complete subspace of $\Omega$. Then $G, H$, and $\ell$ have a unique point of coincidence. In addition, if $(G, \ell)$ and $(H, \ell)$ are weakly-compat$i b l e$, then $G, H$, and $\ell$ have a unique CFP.

If we use identity map instead of the mapping $\ell$, i.e., $\ell=I$ , in Theorem 16, we get the following corollary.

Corollary 19. Let $\left(\Omega, M_{F}, *\right)$ be a complete FM space in which a FM $M_{F}$ is triangular. Let $G, H: \Omega \longrightarrow \Omega$ be a pair of self-mappings and satisfies

$$
\begin{aligned}
& \frac{1}{M_{F}(G \xi, H \eta, t)}-1 \leq \beta_{1}\left(\frac{1}{M_{F}(\xi, \eta, t)}-1\right) \\
& +\beta_{2}\left(\frac{1}{M_{F}(\xi, G \xi, t)}-1\right)+\beta_{3}\left(\frac{1}{M_{F}(\eta, H \eta, t)}-1\right) \\
& +\beta_{4}\left(\frac{1}{M_{F}(\eta, G \xi, t)}-1\right)+\beta_{5}\left(\frac{1}{M_{F}(\xi, H \eta, t)}-1\right) \\
& +\beta_{6}\left(\frac{1}{\aleph(G, H, I, \xi, \eta, t)}-1\right)
\end{aligned}
$$

where

$$
\begin{aligned}
& \frac{1}{\aleph(G, H, I, \xi, \eta, t)}-1=\min \left\{\left(\frac{1}{M_{F}(\xi, G \xi, t)}-1\right),\right. \\
& \left(\frac{1}{M_{F}(\eta, H \eta, t)}-1\right),\left(\frac{1}{M_{F}(\xi, H \eta, t)}-1\right), \\
& \left.\left(\frac{1}{M_{F}(\eta, G \xi, t)}-1\right)\right\},
\end{aligned}
$$

for all $\xi, \eta \in \Omega, t>0$, and $\beta_{1}, \beta_{2}, \beta_{3}, \beta_{4}, \beta_{5}, \beta_{6} \in[0,1)$ with ( $\left.\beta_{1}+\beta_{2}+\beta_{3}+2 \beta_{4}+2 \beta_{5}\right)<1$. Then $G$ and $H$ have a unique CFP.

Example 20. From Example 15, let we define the three-selfmappings $G, H, \ell: \Omega \longrightarrow \Omega$ by: as for each $\xi \in \Omega$,

$$
G \xi=H \xi= \begin{cases}\frac{3}{10}\left(\frac{2 \xi}{7}+\frac{3}{5}\right), & \text { if } \xi \neq 0 \\ 0, & \text { if } \xi=0\end{cases}
$$

And

$$
\ell \xi= \begin{cases}\left(\frac{2 \xi}{7}+\frac{3}{5}\right), & \text { if } \xi \neq 0, \\ 0, & \text { if } \xi=0 .\end{cases}
$$

Since, $G(\Omega) \cup H(\Omega) \subset \ell(\Omega)$, then, we have

$$
\begin{aligned}
& \frac{1}{M_{F}(G \xi, H \eta, t)}-1=\frac{|G \xi-H \eta|}{t}=\frac{3}{10}\left|\frac{2 \xi}{7}-\frac{2 \eta}{7}\right| \\
& =\frac{3}{10}\left(\frac{1}{M_{F}(\ell \xi, \ell \eta, t)}-1\right) \text { for all } \xi, \eta \in \Omega \text { and } t>0
\end{aligned}
$$


which shows that the three self-mappings are weaklycompatible fuzzy-contractive in a complete FM space $(\Omega$, $\left.M_{F}, *\right)$. Thus, all the conditions of Theorem 16 are satisfied with $\beta_{1}=3 / 10, \beta_{2}=\beta_{3}=1 / 5, \beta_{4}=\beta_{5}=2 / 15$, and $\beta_{6}=0$, and the three self-mappings have a unique coincidence point and CFP in $\Omega$, that is, 0 .

\section{Application}

This section deals with the application of the fuzzy differential equations (FDEs) to support our work. From the book of Lakshmikantham and Mohapatra [38], we have the following FDEs.

Let $\mathbb{F}$ be the space of all fuzzy subsets $\xi$ of $\Re$ where $\xi$ $: \mathfrak{R} \longrightarrow[0,1]$. Let the boundary value problem (BVP) is

$$
\begin{gathered}
\xi^{\prime \prime}(\tau)=P\left(\tau, \xi(\tau), \xi^{\prime}(\tau)\right), \quad \tau \in \mathbf{I}=[a, b], \\
\xi\left(\tau_{1}\right)=\xi_{1}, \xi\left(\tau_{2}\right)=\xi_{2}, \quad \tau_{1}, \tau_{2} \in \mathbf{I}=[a, b],
\end{gathered}
$$

where $P: \mathbf{I} \times \mathbb{F} \times \mathbb{F} \longrightarrow \mathbb{F}$ is a continuous function. This problem is equivalent to the integral equation

$$
\xi(\tau)=\int_{\tau_{1}}^{\tau_{2}} Q(\tau, v)\left(P\left(v, \xi(v), \xi^{\prime}(v)\right)\right) d v+\hbar(\tau),
$$

where Green's function $Q$ is given by

$$
Q(\tau, v)= \begin{cases}\frac{\left(\tau_{2}-\tau\right)\left(v-\tau_{1}\right)}{\tau_{2}-\tau_{1}}, & \tau_{1} \leq v \leq \tau \leq \tau_{2}, \\ \frac{\left(\tau_{2}-v\right)\left(\tau-\tau_{1}\right)}{\tau_{2}-\tau_{1}}, & \tau_{1} \leq \tau \leq v \leq \tau_{2} .\end{cases}
$$

And $\hbar(\tau)$ satisfies $\hbar^{\prime \prime}=0, \hbar\left(\tau_{1}\right)=\xi_{1}, \hbar\left(\tau_{2}\right)=\xi_{2}$. Here, we recall some properties of $Q(\tau, v)$, that are

$$
\int_{\tau_{1}}^{\tau_{2}} Q(\tau, v) d v \leq \frac{\left(\tau_{2}-\tau_{1}\right)^{2}}{8}
$$

and

$$
\int_{\tau_{1}}^{\tau_{2}} Q_{\tau}(\tau, v) d v \leq \frac{\tau_{2}-\tau_{1}}{2}
$$

Let $\Omega=\Omega^{1}(\mathbf{I}, \mathbb{F}), *$ is a continuous $t$-norm, and a fuzzy metric $M_{F}: \Omega \times \Omega \times(0, \infty) \longrightarrow[0,1]$ be defined as

$$
M_{F}(\xi, \eta, t)=\frac{t}{t+m(\xi, \eta)} \text { where } m(\xi, \eta)=|\xi-\eta|,
$$

$\forall \xi, \eta \in \Omega$, and $t>0$. Then easily one can prove that $M_{F}$ is triangular and $\left(\Omega, M_{F}, *\right)$ is a complete FM space.

Now, we prove the existing result for the above BVP by using Theorem 11.

Theorem 21. Suppose that $P_{1}, P_{2}: I \times \mathbb{F} \times \mathbb{F} \longrightarrow \mathbb{F}$ and let there exist $\alpha, \beta \in(0,1)$ with $\alpha \leq \beta$ such that for all $\xi, \eta \in \Omega^{1}$
$(I, \mathbb{F})$, satisfies

$$
\begin{aligned}
& \left|P_{1}\left(\tau, \xi(\tau), \xi^{\prime}(\tau)\right)-P_{2}\left(\tau, \eta(\tau), \eta^{\prime}(\tau)\right)\right| \\
& \leq \alpha|\xi(\tau)-\eta(\tau)|+\beta\left|\xi^{\prime}(\tau)-\eta^{\prime}(\tau)\right| .
\end{aligned}
$$

Let there exists $k \in(0,1)$ such that

$m(\xi(\tau), \eta(\tau))=m(\xi, \eta) \leq k \mathbf{D}(G, H, \ell, \xi, \eta), \forall \xi, \eta \in \Omega$,

where

$\mathbf{D}(G, H, \ell, \xi, \eta)=\left\{\begin{array}{c}|\xi-\eta|, \\ \left|\xi-A_{\xi}-\hbar\right|+\left|\eta-B_{\eta}-\hbar\right|+\left|\eta-A_{\xi}-\hbar\right|+\left|\xi-B_{\eta}-\hbar\right| \\ \max \left\{\left|\xi-A_{\xi}-\hbar\right|,\left|\eta-B_{\eta}-\hbar\right|,\left|\eta-A_{\xi}-\hbar\right|,\left|\xi-B_{\eta}-\hbar\right|\right\}\end{array}\right\}$

Then the integral equations

$$
\xi(\tau)=\int_{\tau_{1}}^{\tau_{2}} Q(\tau, v)\left(P_{1}\left(v, \xi(v), \xi^{\prime}(v)\right) d v+\hbar(\tau), \tau \in \mathbf{I},\right.
$$

and

$$
\eta(\tau)=\int_{\tau_{1}}^{\tau_{2}} Q(\tau, v)\left(P_{2}\left(v, \eta(v), \eta^{\prime}(v)\right) d v+\hbar(v), \tau \in \mathbf{I},\right.
$$

have a unique common solution in $\Omega^{1}\left[\left[\tau_{1}, \tau_{2}\right], \mathbb{F}\right]$.

Proof. Suppose that $\Omega=\left[\left[\tau_{1}, \tau_{2}\right], \mathbb{F}\right]$ with metric

$$
m(\xi, \eta)=\max _{\tau_{1} \leq \tau \leq \tau_{2}}\left(\alpha|\xi(\tau)-\eta(\tau)|+\beta\left|\xi^{\prime}(\tau)-\eta^{\prime}(\tau)\right|\right) .
$$

The space $(\Omega, m)$ is a complete metric space. Now, we define the operators $G, H, \ell: \Omega \longrightarrow \Omega$ as

$$
G(\xi)=A_{\xi}+\hbar, H(\eta)=B_{\eta}+\hbar, \ell(\xi)=\xi \text {, and } \ell(\eta)=\eta \text {, }
$$

where

$$
A_{\xi}(\tau)=\int_{\tau_{1}}^{\tau_{2}} Q(\tau, v)\left(P_{1}\left(v, \xi(v), \xi^{\prime}(v)\right) d v, \tau \in \mathbf{I},\right.
$$

and

$$
B_{\eta}(v)=\int_{\tau_{1}}^{\tau_{2}} Q(\tau, v)\left(P_{2}\left(v, \eta(v), \eta^{\prime}(v)\right) d v, \tau \in \mathbf{I},\right.
$$

where $P_{1}, P_{2} \in \Omega(\mathbf{I} \times \mathbb{F} \times \mathbb{F}, \mathbb{F}), \xi, \eta \in \Omega^{1}(\mathbf{I}, \mathbb{F})$, and $\hbar \in \Omega(\mathbf{I}, \mathbb{F})$ . Now by the properties of $Q(\tau, v)$, and from (91), (92) and by using the hypothesis, we have 


$$
\begin{aligned}
& |G(\xi)(\tau)-H(\eta)(\tau)| \leq \int_{\tau_{1}}^{\tau_{2}}|Q(\tau, v)| \mid P_{1}\left(v, \xi(v), \xi^{\prime}(v)\right) \\
& -P_{2}\left(v, \eta(v), \eta^{\prime}(v)\right)\left|d v \leq m(\xi, \eta) \int_{\tau_{1}}^{\tau_{2}}\right| Q(\tau, v) \mid d v \\
& \leq \frac{\left(\tau_{2}-\tau_{1}\right)^{2}}{8} m(\xi, \eta) \leq \frac{m(\xi, \eta)}{8} .
\end{aligned}
$$

And

$$
\begin{aligned}
& \left|(G(\xi))^{\prime}(\tau)-(H(\eta))^{\prime}(\tau)\right| \leq \int_{\tau_{1}}^{\tau_{2}}\left|Q_{\tau}(\tau, v)\right| \mid P_{1}\left(v, \xi(v), \xi^{\prime}(v)\right) \\
& -P_{2}\left(v, \eta(v), \eta^{\prime}(v)\right)\left|d v \leq m(\xi, \eta) \int_{\tau_{1}}^{\tau_{2}}\right| Q_{\tau}(\tau, v) \mid d v \\
& \quad \leq \frac{\tau_{2}-\tau_{1}}{2} m(\xi, \eta) \leq \frac{m(\xi, \eta)}{2} .
\end{aligned}
$$

Now, from the above and by view of (86), and (91), we have that

$$
\begin{aligned}
m(G \xi, H \eta)= & \max _{\tau_{1} \leq \tau \leq \tau_{2}}\left(\alpha|G(\xi)(\tau)-H(\eta)(\tau)|+\beta \mid(G(\xi))^{\prime}(\tau)\right. \\
& \left.-(H(\eta))^{\prime}(\tau) \mid\right) \leq \alpha \frac{m(\xi, \eta)}{8}+\beta \frac{m(\xi, \eta)}{2} \\
\leq & \left(\frac{5}{8} \beta\right) m(\xi, \eta) .
\end{aligned}
$$

Now, from (87), we have that

$$
m(G \xi, H \eta) \leq\left(\frac{5}{8} \beta\right) m(\xi, \eta) \leq Y^{*} \mathbf{D}(G, H, \ell, \xi, \eta), \forall \xi, \eta \in \Omega,
$$

where $Y^{*}=5 / 8 \beta k<1$. Now we are in the position to apply Theorem 11 to get that $G, H$, and $\ell$ have a unique CFP $u^{*} \in$ $\Omega$, i.e., $u^{*}$ is a solution of the BVP. We have the following two main cases:

(a) If $\mathbf{D}(G, H, \ell, \xi, \eta)=|\xi-\eta|$ in (88), then from (85) and (98), for $t>0$, we have

$$
\begin{aligned}
\frac{1}{M_{r}(G \xi, H \eta, t)}-1 & =\frac{m(G \xi, H \eta)}{t} \leq Y^{*} \frac{\mathbf{D}(G, H, \ell, \xi, \eta)}{t} \\
& =Y^{*} \frac{|\xi-\eta|}{t}=Y^{*}\left(\frac{1}{M_{r}(\xi, \eta, t)}-1\right)
\end{aligned}
$$

for all $\xi, \eta \in \Omega$. Hence, the operators $G, H$, and $\ell$ satisfy all the conditions of Theorem 11 with $Y^{*}=\beta_{1}$ and $\beta_{2}=\beta_{3}=0$ in (5). Thus, the operators $G, H$, and $\ell$ have a unique CFP $u^{*} \in \Omega$, i.e., $u^{*}$ is a solution of the BVP (80). (b) If $\mathbf{D}(G, H, \ell, \xi, \eta)=\left|\xi-A_{\xi}-\hbar\right|+\left|\eta-B_{\eta}-\hbar\right|+\mid \eta-$ $A_{\xi}-\hbar|+| \xi-B_{\eta}-\hbar \mid$ in (88), then from (85) and (98), for $t>0$, we have

$$
\begin{aligned}
\frac{1}{M_{r}(G \xi, H \eta, t)}-1=\frac{m(G \xi, H \eta)}{t} \leq Y^{*} \frac{\mathbf{D}(G, H, \ell, \xi, \eta)}{t} \\
=Y^{*} \frac{\left|\xi-A_{\xi}-\hbar\right|+\left|\eta-B_{\eta}-\hbar\right|+\left|\eta-A_{\xi}-\hbar\right|+\left|\xi-B_{\eta}-\hbar\right|}{t} \\
\quad=Y^{*}\left(\frac{|\xi-G \xi|}{t}+\frac{|\eta-H \eta|}{t}+\frac{|\eta-G \xi|}{t}+\frac{|\xi-H \eta|}{t}\right) \\
=Y^{*}\left(\left(\frac{1}{M_{F}(\ell \xi, G \xi, t)}-1\right)+\left(\frac{1}{M_{F}(\ell \eta, H \eta, t)}-1\right)\right. \\
\left.\quad+\left(\frac{1}{M_{F}(\ell \eta, G \xi, t)}-1\right)+\left(\frac{1}{M_{F}(\ell \xi, H \eta, t)}-1\right)\right),
\end{aligned}
$$

for all $\xi, \eta \in \Omega$. Hence, the operators $G, H$, and $\ell$ satisfy all the conditions of Theorem 11 with $Y^{*}=\beta_{2}$ and $\beta_{1}=\beta_{3}=0$ in (5). Thus, the operators $G, H$, and $\ell$ have a unique CFP $u^{*} \in \Omega$, i.e., $u^{*}$ is a solution of the BVP (80).

(c) If $\mathbf{D}(G, H, \ell, \xi, \eta)=\max \left\{\left|\xi-A_{\xi}-\hbar\right|,\left|\eta-B_{\eta}-\hbar\right|, \mid \eta\right.$ $-A_{\xi}-\hbar|,| \xi-B_{\eta}-\hbar \mid$ in (88), then, we may have the following four subcases.

(i) If $\left|\xi-A_{\xi}-\hbar\right|$ is the maximum term in $\left\{\mid \xi-A_{\xi}\right.$ $\left.-\hbar|,| \eta-B_{\eta}-\hbar|,| \eta-A_{\xi}-\hbar|,| \xi-B_{\eta}-\hbar \mid\right\}$. Then, $\mathbf{D}(G, H, \ell, \xi, \eta)=\left|\xi-A_{\xi}-\hbar\right|$ in (88). Now from (85) and (98), for $t>0$, we have

$$
\begin{aligned}
& \frac{1}{M_{r}(G \xi, H \eta, t)}-1=\frac{m(G \xi, H \eta)}{t} \leq Y^{*} \frac{\mathbf{D}(G, H, \ell, \xi, \eta)}{t} \\
& =Y^{*} \frac{\left|\xi-A_{\xi}-\hbar\right|}{t}=Y^{*} \frac{|\xi-G \xi|}{t} \\
& \quad=Y^{*}\left(\frac{1}{M_{r}(\xi, G \xi, t)}-1\right), \text { for all } \xi, \eta \in \Omega .
\end{aligned}
$$

(ii) If $\left|\eta-B_{\eta}-\hbar\right|$ is the maximum term in $\left\{\left|\xi-A_{\xi}-\hbar\right|\right.$ , $\left.\left|\eta-B_{\eta}-\hbar\right|,\left|\eta-A_{\xi}-\hbar\right|,\left|\xi-B_{\eta}-\hbar\right|\right\}$. Then, $\mathbf{D}(G$, $H, \ell, \xi, \eta)=\left|\eta-B_{\eta}-\hbar\right|$ in (88). Now from (85) and (98), for $t>0$, we have

$$
\begin{aligned}
& \frac{1}{M_{r}(G \xi, H \eta, t)}-1=\frac{m(G \xi, H \eta)}{t} \leq Y^{*} \frac{\mathbf{D}(G, H, \ell, \xi, \eta)}{t} \\
& =Y^{*} \frac{\left|\eta-B_{\eta}-\hbar\right|}{t}=Y^{*} \frac{|\eta-H \eta|}{t} \\
& =Y^{*}\left(\frac{1}{M_{r}(\eta, H \eta, t)}-1\right), \text { for all } \xi, \eta \in \Omega .
\end{aligned}
$$

(iii) If $\left|\eta-A_{\xi}-\hbar\right|$ is the maximum term in $\left\{\mid \xi-A_{\xi}\right.$ $\left.-\hbar|,| \eta-B_{\eta}-\hbar|,| \eta-A_{\xi}-\hbar|,| \xi-B_{\eta}-\hbar \mid\right\}$. Then, 
$\mathbf{D}(G, H, \ell, \xi, \eta)=\left|\eta-A_{\xi}-\hbar\right|$ in (88). Now from (85) and (98), for $t>0$, we have

$$
\begin{aligned}
& \frac{1}{M_{r}(G \xi, H \eta, t)}-1=\frac{m(G \xi, H \eta)}{t} \leq Y^{*} \frac{\mathbf{D}(G, H, \ell, \xi, \eta)}{t} \\
& =Y^{*} \frac{\left|\eta-A_{\xi}-\hbar\right|}{t}=Y^{*} \frac{|\eta-G \xi|}{t} \\
& =Y^{*}\left(\frac{1}{M_{r}(\eta, G \xi, t)}-1\right), \text { for all } \xi, \eta \in \Omega .
\end{aligned}
$$

(iv) If $\left|\xi-B_{\eta}-\hbar\right|$ is the maximum term in $\left\{\mid \xi-A_{\xi}-\hbar\right.$ $\left.|,| \eta-B_{\eta}-\hbar|,| \eta-A_{\xi}-\hbar|,| \xi-B_{\eta}-\hbar \mid\right\}$. Then, $\mathbf{D}(G$ $, H, \ell, \xi, \eta)=\left|\xi-B_{\eta}-\hbar\right|$ in (88). Now from (98) and (98), for $t>0$, we have

$$
\begin{aligned}
& \frac{1}{M_{r}(G \xi, H \eta, t)}-1=\frac{m(G \xi, H \eta)}{t} \leq Y^{*} \frac{\mathbf{D}(G, H, \ell, \xi, \eta)}{t} \\
& =Y^{*} \frac{\left|\xi-B_{\eta}-\hbar\right|}{t}=Y^{*} \frac{\left|\xi-B_{\eta}-\hbar\right|}{t} \\
& =Y^{*}\left(\frac{1}{M_{r}(\xi, H \eta, t)}-1\right), \text { for all } \xi, \eta \in \Omega .
\end{aligned}
$$

for all $\xi, \eta \in \Omega$. Hence, from (i)-(vi), the operators $G, H$, and $\ell$ satisfy all the conditions of Theorem 11 with $Y^{*}=\beta_{3}$ and $\beta_{1}=\beta_{2}=0$ in (5). Thus, the operators $G, H$, and $\ell$ have a unique CFP $u^{*} \in \Omega$, i.e., $u^{*}$ is a solution of the BVP (80)

\section{Conclusion}

In this paper, we proved some generalized unique coincidence points and CFP theorems for weakly-compatible three self-mappings in FM spaces without the assumption that the "fuzzy contractive sequences are Cauchy." The "triangular property of FM" is used as a basic tool throughout the complete paper to get the existence of unique coincidence points and CFP results in FM spaces. In support of our main work, we presented two illustrative examples, that is, Examples 15 and 20. In addition, we established an application of fuzzy differential equations to ensure the existence of a unique common solution to support our work. By using this concept, one can contribute different contractive types of FP, CFP, and coincidence points result in FM spaces with different types of applications.

\section{Data Availability}

Data sharing is not applicable to this article as no data set were generated or analyzed during the current study.

\section{Conflicts of Interest}

The authors declare that there is no conflict of interest regarding the publication of this paper.

\section{Acknowledgments}

The authors are grateful to the Deanship of Scientific Research, King Saud University for funding through Vice Deanship of Scientific Research Chairs.

\section{References}

[1] L. A. Zadeh, "Fuzzy sets," Information and Control, vol. 8, no. 3, pp. 338-353, 1965.

[2] O. Kramosil and J. Michalek, "Fuzzy metric and statistical metric spaces," Kybernetika, vol. 11, pp. 336-344, 1975.

[3] A. George and P. Veeramani, "On some results in fuzzy metric spaces,” Fuzzy Sets and Systems, vol. 64, no. 3, pp. 395-399, 1994.

[4] M. Grabiec, "Fixed points in fuzzy metric spaces," Fuzzy Sets and Systems, vol. 27, no. 3, pp. 385-389, 1988.

[5] V. Gregori and A. Sapena, "On fixed-point theorems in fuzzy metric spaces," Fuzzy Sets and Systems, vol. 125, no. 2, pp. 245-252, 2002.

[6] M. Imdad and J. Ali, "Some common fixed point theorems in fuzzy metric spaces," Mathematical Communications, vol. 11, pp. 153-163, 2006.

[7] D. Mihet, "A Banach contraction theorem in fuzzy metric spaces," Fuzzy Sets and Systems, vol. 144, no. 3, pp. 431-439, 2004.

[8] D. Mihet, "A class of contractions in fuzzy metric spaces," Fuzzy Sets and Systems, vol. 161, no. 8, pp. 1131-1137, 2010.

[9] C. D. Bari and C. Vetro, "Fixed points, attractors and weak fuzzy contractive mappings in a fuzzy metric space," Journal of Fuzzy Mathematics, vol. 1, pp. 973-982, 2005.

[10] T. Som, "Some results on common fixed point in fuzzy metric spaces," Soochow Journal of Mathematics, vol. 33, pp. 553-561, 2007.

[11] A. Aliouche, F. Merghadi, and A. Djoudi, "A related fixed point theorem in two fuzzy metric spaces," Journal of Nonlinear Sciences and Applications, vol. 2, no. 1, pp. 19-24, 2009.

[12] K. P. R. Rao, A. Aliouche, and G. R. Babu, "Related fixed point theorems in fuzzy metric spaces," Journal of Nonlinear Sciences and Applications, vol. 1, no. 3, pp. 194-202, 2008.

[13] O. Hadzic and E. Pap, "A fixed point theorem for multivalued mappings in probabilistic metric spaces and an application in fuzzy metric spaces," Fuzzy Sets and Systems, vol. 127, no. 3, pp. 333-344, 2002.

[14] F. Kiany and A. Amini-Haradi, "Fixed point and endpoint theorems for set-valued fuzzy contraction maps in fuzzy metric spaces," Fixed Point Theory and Applications, vol. 2011, no. 1, 2011.

[15] I. Beg, S. Sedghi, and N. Shobe, "Fixed point theorems in fuzzy metric spaces," International Journal of Analysis, vol. 2013, Article ID 934145, 4 pages, 2013.

[16] A. F. Roldán-López-de-Hierro, E. Karapınar, and S. Manro, "Some new fixed point theorems in fuzzy metric spaces," Journal of Intelligent Fuzzy Systems, vol. 27, no. 5, pp. 2257-2264, 2014. 
[17] M. Jleli, E. Karapinar, and B. Samet, "On cyclic $(\psi, \phi)$-contractions in Kaleva-Seikkala's type fuzzy metric spaces," Journal of Intelligent Fuzzy Systems, vol. 27, no. 4, pp. 2045-2053, 2014.

[18] X. Li, S. U. Rehman, S. U. Khan, H. Aydi, J. Ahmad, and N. Hussain, "Strong coupled fixed point results and applications to Urysohn integral equations," Dynamic Systems and Applications, vol. 30, pp. 197-218, 2021.

[19] S. U. Rehman, R. Chinram, and C. Boonpok, "Rational type fuzzy-contraction results in fuzzy metric spaces with an application," Journal of Mathematics, vol. 2021, Article ID 6644491, 13 pages, 2021.

[20] I. Shamas, S. U. Rehman, H. Aydi, T. Mahmood, and E. Ameer, "Unique fixed-point results in fuzzy metric spaces with an application to Fredholm integral equations," Journal of Function Spaces, vol. 2021, Article ID 4429173, 12 pages, 2021.

[21] S. Jabeen, S. U. Rehman, Z. Zheng, and W. Wei, "Weakly compatible and quasi-contraction results in fuzzy cone metric spaces with application to the Urysohn type integral equations," Advances in Differential Equations, vol. 2020, no. 1, p. 280,2020

[22] A. Fulga, H. Afshari, and H. Shojaat, "Common fixed point theorems on quasi-cone metric space over a divisible Banach algebra," Advances in Differential Equations, vol. 2021, no. 1, pp. 1-15, 2021.

[23] H. Aydi, E. Karapinar, and Z. Mustafa, "Coupled coincidence point results on generalized distance in ordered cone metric spaces," Positivity, vol. 17, no. 4, pp. 979-993, 2013.

[24] R. H. Haghi, S. H. Rezapour, and N. Shahzad, "Some fixed point generalizations are not real generalizations," Nonlinear Analysis, vol. 74, no. 5, pp. 1799-1803, 2011.

[25] M. Imdad, J. Ali, and M. Tanveer, "Coincidence and common fixed point theorems for nonlinear contractions in menger PM spaces," Chaos, Solitons \& Fractals, vol. 42, no. 5, pp. 31213129, 2009.

[26] E. Karapinar and U. Yuksel, "On common fixed point theorems without commuting conditions in TVS-cone metric spaces," Journal of Computational Analysis and Applications, vol. 13, pp. 1115-1122, 2011.

[27] A. Latif, N. Hussain, and J. Ahmad, "Coincidence points for hybrid contractions in cone metric spaces," Journal of Nonlinear and Convex Analysis, vol. 17, pp. 899-906, 2016.

[28] Y. Liu and Z. Li, "Coincidence point theorems in probabilistic and fuzzy metric spaces," Fuzzy Sets and Systems, vol. 158, no. 1, pp. 58-70, 2007.

[29] J. Mathuraiveera and S. Mookia, "Commonfixed point theorems in M-fuzzy cone metric space," Results in Nonlinear Analysis, vol. 4, no. 2021, pp. 33-46, 2021.

[30] B. D. Pant and S. Chauhan, "Common fixed point theorems for two pairs of weakly compatible mappings in menger spaces and fuzzy metric spaces," Scientific Studies and Research, vol. 21, pp. 81-96, 2011.

[31] S. U. Rehman, S. Jabeen, F. Abbas, H. Ullah, and I. Khan, "Common fxed point theorems for compatible and weakly compatible maps in fuzzy cone metric spaces," Annals of Fuzzy Mathematics and Informatics, vol. 19, no. 1, pp. 1-19, 2020.

[32] P. Saha, B. S. Choudhury, and P. Das, "Weak coupled coincidence point results having a partially ordering in fuzzy metric spaces," Fuzzy Information and Engineering, vol. 8, no. 2, pp. 199-216, 2016.
[33] W. Shatanawi, E. Karapinar, and H. Aydi, "Coupled coincidence points in partially ordered cone metric spaces with a cdistance," Journal of Applied Mathematics, vol. 2012, Article ID 312078, 15 pages, 2012.

[34] E. Karapinar, P. Kumam, and W. Sintunavarat, "Coupled fixed point theorems in cone metric spaces with a c-distance and applications," Fixed Point Theory and Applications, vol. 2012, no. 1, p. 19, 2012.

[35] S. Shukla, S. K. Malhotra, and P. Bhargava, "Ordered weak contractions in cone metric spaces over Banach algebras and fixed point theorems," Advances in the Theory of Nonlinear Analysis and its Application, vol. 3, pp. 102-110, 2019.

[36] B. Schweizer and A. Sklar, "Statical metric spaces," Pacific Journal of Mathematics, vol. 10, pp. 314-334, 1960.

[37] M. Abbas and G. Jungck, "Common fixed point results for noncommuting mappings without continuity in cone metric spaces," Journal of Mathematical Analysis and Applications, vol. 341, no. 1, pp. 416-420, 2008.

[38] V. Lakshmikantham and R. Mohapatra, Theory of Fuzzy Differential Equations and Inclusion, Taylor and Francis, London, 2003. 